\title{
Article \\ Modeling and Design of High-Power Enhanced Leakage- Inductance-Integrated Medium-Frequency Transformers for DAB Converters
}

\author{
Xinsheng Zhang (D, Fei Xiao, Ruitian Wang *, Wei Kang and Beichao Yang
}

check for updates

Citation: Zhang, X.; Xiao, F.; Wang, R.; Kang, W.; Yang, B. Modeling and Design of High-Power Enhanced Leakage-Inductance-Integrated Medium-Frequency Transformers for DAB Converters. Energies 2022, 15, 1361. https://doi.org/10.3390/ en15041361

Academic Editors: Alon Kuperman and Alessandro Lampasi

Received: 27 January 2022

Accepted: 10 February 2022

Published: 14 February 2022

Publisher's Note: MDPI stays neutral with regard to jurisdictional claims in published maps and institutional affiliations.

Copyright: (C) 2022 by the authors. Licensee MDPI, Basel, Switzerland. This article is an open access article distributed under the terms and conditions of the Creative Commons Attribution (CC BY) license (https:// creativecommons.org/licenses/by/ $4.0 /)$.

\author{
National Key Laboratory of Science and Technology on Vessel Integrated Power System, Naval University \\ of Engineering, Wuhan 430033, China; marvinzxs@163.com (X.Z.); xfeyninger@gmail.com (F.X.); \\ kangxiaodouXJTU@163.com (W.K.); yangbeichao199405@163.com (B.Y.) \\ * Correspondence: wangrt4321@163.com
}

\begin{abstract}
For dual active bridge (DAB) converters, integrating the phase-shifting inductance (PSI) in the medium-frequency transformer (MFT) is an effective way to improve the overall power density. Different from the existing leakage-inductance-integrated (LII) structure, a concentricwinding (CW) enhanced leakage-inductance-integrated (ELII) structure, which includes an additional core, is proposed in this paper. In order to explain the operating mode of CW ELII MFT, a magnetic circuit model is established, and the analysis is carried out under the typical DAB excitation. The total leakage inductance of CW ELII MFT is divided into the winding leakage inductance and the additional leakage inductance for calculation. The integrated structure makes the heat dissipation of the MFT challenging. Therefore, the air-water combined cooling method is adopted in the design. A thermal resistance model is built for the winding air channel under forced convection. On this basis, MFT designs with different integration structures for different leakage inductance requirements are compared. Finally, a $200 \mathrm{~kW} / 4 \mathrm{kHz} / 200 \mu \mathrm{H}$ MFT prototype was designed and manufactured, which achieved the power density of $5.16 \mathrm{~kW} / \mathrm{dm}^{3}$ and the efficiency of $99.30 \%$. The prototype was tested in a DAB converter, which is a module of a $2 \mathrm{MW}$ modular multilevel converter-bidirectional DC-DC converter (MMC-BDC).
\end{abstract}

Keywords: medium-frequency transformer; inductor integration; DAB converter; optimal design

\section{Introduction}

In recent years, benefiting from advances in switching devices, magnetic materials, and control methods, power conversion systems based on MFTs have gradually increased. With the increase of operating frequency, the volume and weight of the transformer can be reduced, thus improving the power density of the converter. Therefore, the MFT has broad application prospects in many fields-such as flexible DC transmission, renewable energy grid connection, and electrified transportation [1-3]. A typical application occasion is vessel integrated power system (IPS) [4], and a modular multi-level converter-bidirectional DC/DC converter (MMC-BDC) for IPS is shown in Figure 1 [5]. The converter adopts multiple DAB converters to realize DC/DC energy conversion and bidirectional flow, which can serve DC loads or energy storage elements.

In the topology of DAB, MFT provides voltage matching and galvanic isolation. The phase-shifting inductance (PSI) is determined by the power capacity of the DAB converter and provides the instantaneous energy storage in the conversion process [6]. In the reported high-power DAB prototypes, an auxiliary inductor and an MFT is usually used. The volume and weight of the auxiliary inductor may even be close to the MFT [3]. In [7], the integrated/non-integrated MFT designs are compared. Although the integrated design has obvious advantages in power density, the non-integrated design is finally chosen for the sake of heat dissipation and installation flexibility. In [8-10], the design method for 
leakage-inductance-integrated (LII) MFT has been investigated. However, in order to achieve a larger leakage inductance, the LII MFT requires more winding turns or a greater primary-secondary distance, and the unconstrained leakage flux will cause additional losses in tape wound cores. Therefore, it is hard for the LII MFT to meet the requirements of the high inductance and the high power.

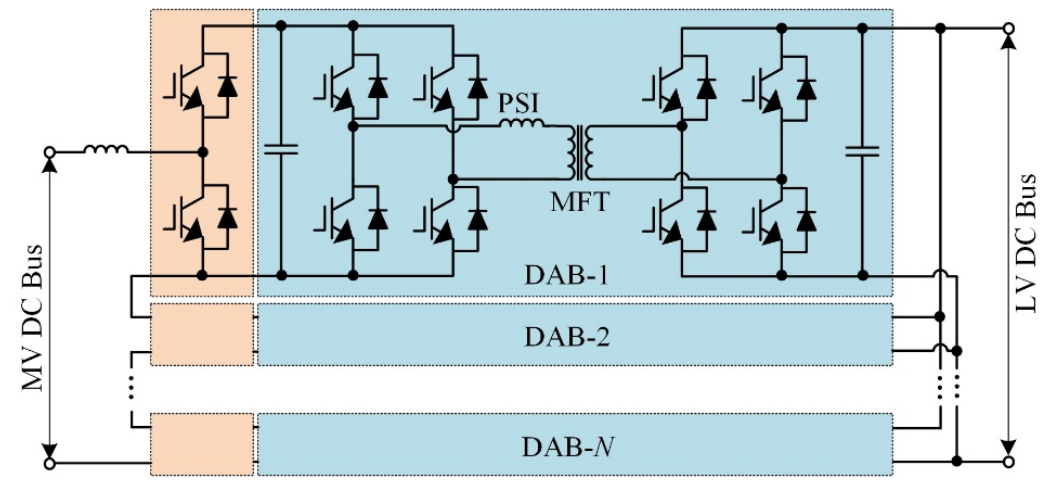

Figure 1. The modular multilevel converter-bidirectional DC-DC converter.

Thermal performance is one of the decisive factors for the success of MFT design. Natural air cooling is the most common heat dissipation method for MFTs. In [11] and [12], the thermal network modeling method for natural air-cooled MFT is comprehensively described. In order to increase power density, water cooling is adopted in [13] and [14], and forced air cooling is adopted in [3,15]. In [3], the forced convection formula for circular tubes is adopted for thermal modeling without considering the structural characteristics of the winding air channel.

Many achievements have emerged in the optimal design of MFT in recent years, which further pushes the MFT to large-scale industrial applications. In [16,17], the brute force grid search method is adopted to optimize the design of a $100 \mathrm{~kW} / 10 \mathrm{kHz}$ shell-type MFT. The sensitivity analysis of design parameters is carried out based on the large-scaled calculation data. In [18], the researchers attempt to establish a unified mathematical model for transformer/inductor optimal design based on existing classical parameter models and carried out the optimal design with the goal of minimum core and minimum loss. A $200 \mathrm{kVA} / 10 \mathrm{kHz}$ shell-type MFT is designed based on the genetic algorithm in [10], and the optimal design of the heat sink is embedded in the design process. The above work is carried out for typical core-type or shell-type MFT. The design of inductor-integrated MFT is more challenging due to the parameter coupling and the integration structure.

Compared with the previous work, the main contributions of the work in this paper include the following: (1) Different from the conventional leakage-inductance-integrated (LII) MFT, a concentric-winding enhanced leakage-inductance-integrated (CW ELII) MFT, which includes an additional core, is proposed in this paper. Moreover, a developed example of $200 \mathrm{~kW}, 4 \mathrm{kHz}, 200 \mu \mathrm{H}$ MFT with CW ELII structure is presented specifically. (2) The air channel in the forced air-cooled winding is simplified to the parallel-plate structure, and the thermal resistance model is established based on classic models. (3) For different leakage inductance requirements, the power density of the CW LII and CW ELII solutions are quantitatively compared.

This paper is organized as follows. Section 2 discusses different integration structures and focuses on the magnetic circuit analysis of the CW ELII MFT in DAB. Section 3 introduces the calculation method of the loss and the leakage inductance. Section 4 presents the thermal modeling method of air-water combined cooled MFT. In Section 5, the design method and quantitative comparison of the CW LII and CW ELII MFT are presented. The developed $200 \mathrm{~kW}, 4 \mathrm{kHz}, 200 \mu \mathrm{H}$ MFT prototype, and experimental results are also presented in Section 5. Finally, Section 6 lays out the conclusions and future work possibilities. 


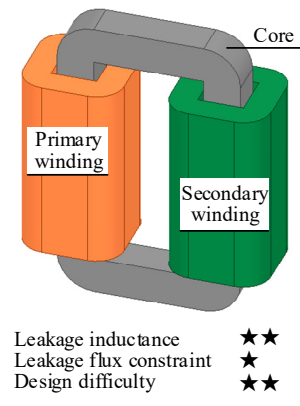

(a)

\section{Integration Structures and Operating Mode}

\subsection{Integration Structures}

The inductor-integrated MFT can be divided into three categories: detached-inductor integrated (DII), leakage-inductance-integrated (LII), and enhanced leakage-inductanceintegrated (ELII) MFT. The characteristic of DII MFT is that it has independent inductor windings. The inductor core and the transformer core are integrated or just packaged together. The DII MFT will not be focused on in this paper.

LII MFT features no additional winding or core. The target leakage inductance is achieved only through the design of the winding structure. Figure $2 \mathrm{a}, \mathrm{b}$ show typical separate-winding (SW) and concentric-winding (CW) structures. It is usually easier to achieve a greater leakage inductance by the SW structure [3]. However, since the magnetic motive force (MMF) on the core limb of the SW MFT is unbalanced, the leakage flux is widely distributed in the external space, which may lead to eddy losses in the adjacent metal structure. Therefore, the SW MFT should be used in compact high-power converters with caution. No matter the SW or the CW structure, part of the leakage flux will pass through the core tape vertically and cause apparent eddy current loss of the wound core [19].

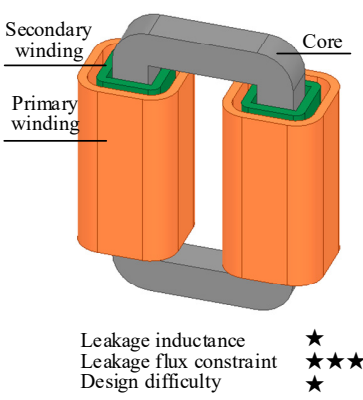

(b)

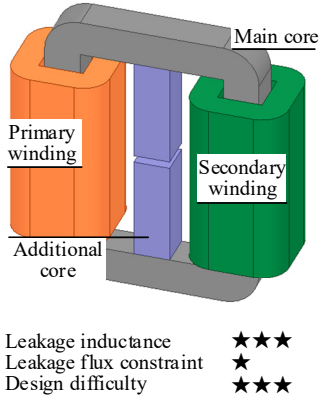

(c)

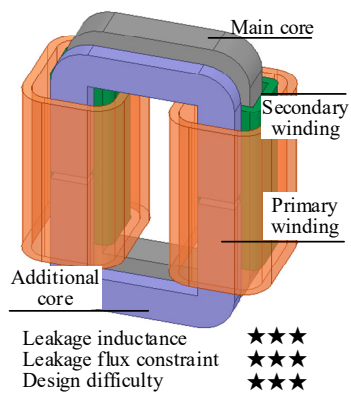

(d)
Figure 2. Comparison of different integration structures. (a) SW LII, (b) CW LII, (c) SW ELII, (d) CW ELII.

The characteristic of ELII MFT is that the additional core is used instead of the air in the leakage flux path of the LII MFT. Due to the higher equivalent permeability of the additional core, it is possible to achieve a higher power density. Figure 2c shows the SW ELII structure, which will cause the external leakage flux like the SW LII structure. The flux from the main core to the additional core limb will pass through the core tape vertically, which will cause local additional core loss [19]. Figure 2d shows the CW ELII structure, in which the additional independent core restricts the flow path of the leakage flux. Therefore, the CW ELII structure may be more suitable for the requirements of the high power and high inductance.

\subsection{CW ELII MFT Operating Mode in DAB}

The circuit topology of DAB is shown in Figure 3. The MFT is excited by H-bridges on both sides simultaneously, and the operating condition is determined by the duty cycle and the phase-shifting duty of the two bridges. In order to analyze the operating mode of the CW ELII MFT under typical DAB excitation, the magnetic circuit model is established. As shown in Figure 4 , the voltage excitations $u_{\mathrm{p}}$ and $u_{\mathrm{s}}$ at the primary and the secondary sides work as flux sources $\Phi_{\mathrm{p}}$ and $\Phi_{\mathrm{S}}$ in the same direction in the magnetic circuit.

$$
\left\{\begin{aligned}
N_{\mathrm{p}} \Phi_{\mathrm{p}}(t) & =\int u_{\mathrm{p}}(t) \mathrm{d} t \\
N_{\mathrm{s}} \Phi_{\mathrm{s}}(t) & =\int u_{\mathrm{s}}(t) \mathrm{d} t
\end{aligned}\right.
$$

where $N_{\mathrm{p}}$ and $N_{\mathrm{s}}$ are the number of turns of the primary and secondary windings. 


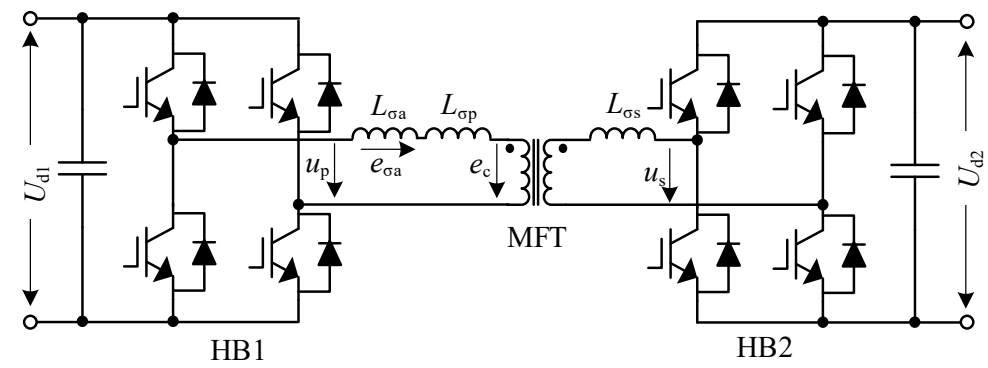

Figure 3. DAB circuit topology.

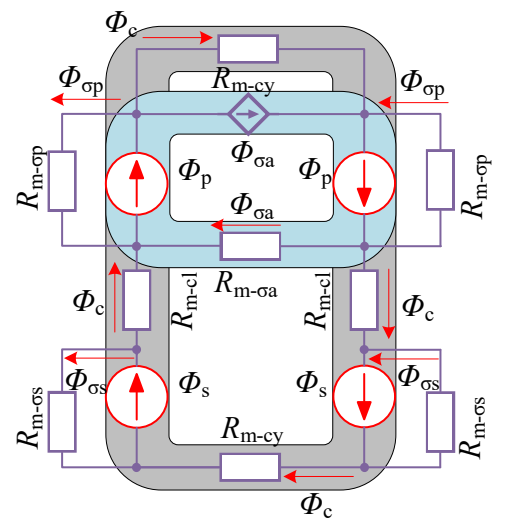

Figure 4. Magnetic circuit model of CW ELII MFT.

In Figure $4, R_{\mathrm{m}-\mathrm{cl}}$ and $R_{\mathrm{m} \text {-cy }}$ are respectively the reluctance of the core limb and core yoke; $R_{\mathrm{m} \text { - op }}$ and $R_{\mathrm{m} \text { - } \sigma \mathrm{s}}$ are respectively the air leakage reluctance of the primary winding and the secondary winding; $R_{\mathrm{m}-\sigma \mathrm{a}}$ is the reluctance of the additional core. The magnetic circuit equation is given as

$$
\left\{\begin{array}{l}
\Phi_{\sigma \mathrm{a}} R_{\mathrm{m}-\sigma \mathrm{a}}-2 \Phi_{\sigma \mathrm{p}} R_{\mathrm{m}-\sigma \mathrm{p}}+\Phi_{\mathrm{c}} R_{\mathrm{m}-\mathrm{cy}}=0 \\
\Phi_{\sigma \mathrm{a}} R_{\mathrm{m}-\sigma \mathrm{a}}-2 \Phi_{\mathrm{c}} R_{\mathrm{m}-\mathrm{cl}}+2 \Phi_{\sigma \mathrm{s}} R_{\mathrm{m}-\sigma \mathrm{s}}-\Phi_{\mathrm{c}} R_{\mathrm{m}-\mathrm{cy}}=0 \\
\Phi_{\sigma \mathrm{a}}+\Phi_{\sigma \mathrm{p}}+\Phi_{\mathrm{c}}=\Phi_{\mathrm{p}} \\
\Phi_{\sigma \mathrm{s}}+\Phi_{\mathrm{c}}=\Phi_{\mathrm{s}}
\end{array}\right.
$$

According to the reluctances, the additional leakage inductance $L_{\sigma a}$, primary leakage inductance $L_{\sigma p}$, and secondary leakage inductance $L^{\prime}{ }_{\sigma s}$ (in primary) can be defined as

$$
\left\{\begin{array}{l}
L_{\sigma \mathrm{a}}=N_{\mathrm{p}}^{2} / R_{\mathrm{m}-\sigma \mathrm{a}} \\
L_{\sigma \mathrm{p}}=N_{\mathrm{p}}^{2} / 2 R_{\mathrm{m}-\sigma \mathrm{p}} \\
L_{\sigma \mathrm{s}}^{\prime}=N_{\mathrm{p}}^{2} / 2 R_{\mathrm{m}-\sigma \mathrm{s}}
\end{array}\right.
$$

Since the main core is without air gaps, the reluctances $R_{\mathrm{m}-\mathrm{cl}}$ and $R_{\mathrm{m} \text {-cy }}$ are much smaller than those of the air or the additional core and can be assumed to be zero. Thus, according to (1) and (2), the magnetic fluxes can be obtained.

$$
\left\{\begin{array}{l}
\Phi_{\mathrm{c}}=\frac{L_{\sigma \mathrm{s}}^{\prime} \Phi_{\mathrm{p}}+\left(L_{\sigma \mathrm{p}}+L_{\sigma \mathrm{a}}\right) \Phi_{\mathrm{s}}}{L_{\sigma \mathrm{s}}^{\prime}+L_{\sigma \mathrm{p}}+L_{\sigma \mathrm{a}}} \\
\Phi_{\sigma \mathrm{a}}=\frac{L_{\sigma \mathrm{a}}\left(\Phi_{\mathrm{p}}-\Phi_{\mathrm{s}}\right)}{L_{\sigma \mathrm{s}}^{\prime}+L_{\sigma \mathrm{p}}+L_{\sigma \mathrm{a}}} \\
\Phi_{\sigma \mathrm{p}}=\frac{L_{\sigma \mathrm{p}}\left(\Phi_{\mathrm{p}}-\Phi_{\mathrm{s}}\right)}{L_{\sigma \mathrm{s}}^{\prime}+L_{\sigma \mathrm{p}}+L_{\sigma \mathrm{a}}} \\
\Phi_{\sigma \mathrm{s}}=\frac{-L_{\sigma \mathrm{s}}^{\prime}\left(\Phi_{\mathrm{p}}-\Phi_{\mathrm{s}}\right)}{L_{\sigma \mathrm{s}}^{\prime}+L_{\sigma \mathrm{p}}+L_{\sigma \mathrm{a}}}
\end{array}\right.
$$


Take typical DAB excitation as an example: the duty of two H-bridges $D_{\mathrm{h}}=0.5$; phaseshifting duty $0<D_{\mathrm{s}}<0.5$. Then the voltage excitations of MFT, $u_{\mathrm{p}}$ and $u_{\mathrm{s}}$, are given as

$$
\begin{aligned}
& u_{\mathrm{p}}(t)=\left\{\begin{array}{c}
U_{\mathrm{d} 1},[0,0.5 T] \\
-U_{\mathrm{d} 1},[0.5 T, T]
\end{array}\right. \\
& u_{\mathrm{s}}(t)=\frac{U_{\mathrm{d} 2}}{U_{\mathrm{d} 1}} u_{\mathrm{p}}\left(t-D_{\mathrm{s}} T\right)
\end{aligned}
$$

where $U_{\mathrm{d} 1}$ is the DC bus voltage of the primary side, and $U_{\mathrm{d} 2}$ is the DC bus voltage of the secondary side.

The induced electromotive force $e_{\mathrm{c}}(t)$ of the main core and $e_{\sigma \mathrm{a}}(t)$ of the additional core can be calculated according to Equations (1), (4), and (5).

$$
\begin{aligned}
& -e_{\mathrm{c}}(t)=\left\{\begin{array}{l}
\frac{L_{\sigma \mathrm{s}}^{\prime}-L_{\sigma \mathrm{p}}-L_{\sigma \mathrm{a}}}{L_{\sigma \mathrm{s}}+L_{\sigma \mathrm{p}}+L_{\sigma \mathrm{a}}} U_{\mathrm{d} 1,},\left[0, D_{\mathrm{s}} T\right] \\
U_{\mathrm{d} 1,}\left[D_{\mathrm{s}} T, 0.5 T\right] \\
\frac{-L_{\sigma \mathrm{s}}^{\prime}+L_{\sigma \mathrm{p}}+L_{\sigma \mathrm{a}}}{L_{\mathrm{\sigma s}}^{\prime}+L_{\sigma \mathrm{p}}+L_{\sigma \mathrm{a}}} U_{\mathrm{d} 1,},\left[0.5 T,\left(0.5+D_{\mathrm{s}}\right) T\right] \\
-U_{\mathrm{d} 1,},\left[\left(0.5+D_{\mathrm{s}}\right) T, T\right]
\end{array}\right. \\
& -e_{\sigma a}(t)=\left\{\begin{array}{l}
\frac{2 L_{\sigma a}}{L_{\sigma \mathrm{s}}^{\prime}+L_{\sigma \mathrm{p}}+L_{\sigma \mathrm{\sigma a}}} U_{\mathrm{d} 1},\left[0, D_{\mathrm{s}} T\right] \\
0,\left[D_{\mathrm{s}} T, 0.5 T\right] \\
-\frac{2 L_{\sigma a}}{L_{\sigma \mathrm{s}}^{\prime}+L_{\sigma \mathrm{p}}+L_{\sigma a}} U_{\mathrm{d} 1},\left[0.5 T,\left(0.5+D_{\mathrm{s}}\right) T\right] \\
0,\left[\left(0.5+D_{\mathrm{s}}\right) T, T\right]
\end{array}\right.
\end{aligned}
$$

For example, the turns ratio of the MFT satisfies $N_{\mathrm{p}}: N_{\mathrm{s}}=U_{\mathrm{d} 1}: U_{\mathrm{d} 2}$, and the leakage inductances satisfies $L_{\sigma \mathrm{a}}+L_{\sigma p}+L_{\sigma s}^{\prime}=5 L_{\sigma p}=5 L_{\sigma s}^{\prime}$. Then the voltage and flux waveforms with the phase-shifting duty of 0 and 0.25 are shown in Figure 5 .
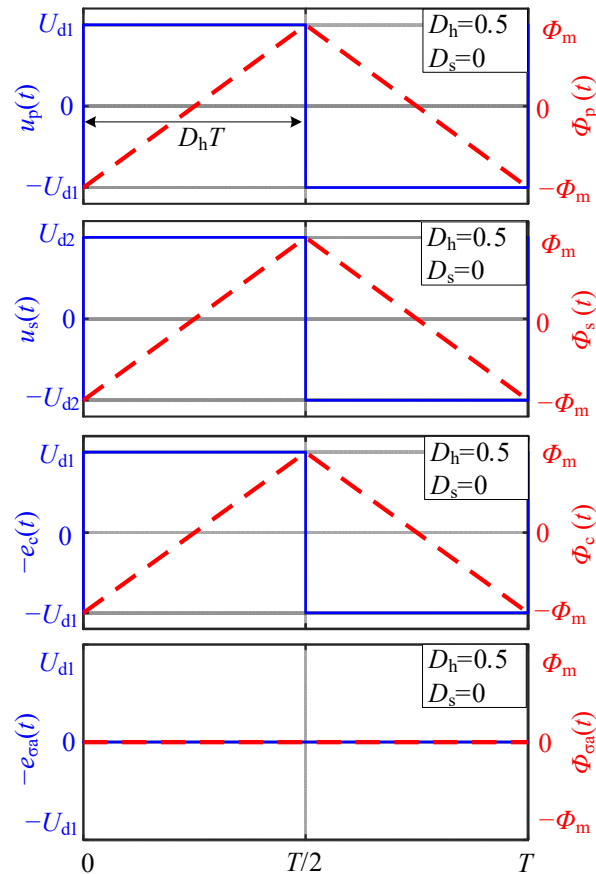

(a)
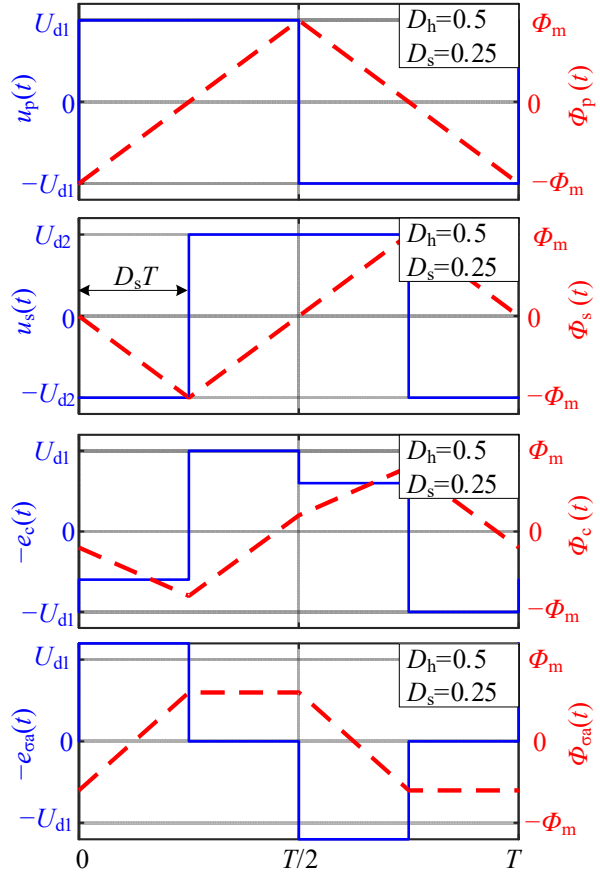

(b)

Figure 5. Voltage, induced electromotive force, and flux waveforms of CW ELII MFT under typical DAB excitation. (a) $D_{\mathrm{h}}=0.5, D_{\mathrm{s}}=0 ;$ (b) $D_{\mathrm{h}}=0.5, D_{\mathrm{s}}=0.25$. 


\section{Loss and Leakage Inductance Calculation}

Loss and inductance are important electrical parameters of MFT. In view of the structure particularity of the CW ELII MFT, this section discusses the calculation methods of the loss and the leakage inductance based on the magnetic circuit analysis above.

\subsection{Core Loss of CW ELII MFT}

The core loss of CW ELII MFT contains the main core loss and the additional core loss. Core loss under non-sinusoidal excitation can be calculated by the improved generalized Steinmetz equation (iGSE) [20], in which the peak value and the differential of the flux density are needed. Based on the magnetic circuit analysis and the assumption that $L_{\sigma p}=L_{\sigma s}^{\prime}$, the peak value and the differential of the flux densities can be derived from (6) and (7) as

$$
\begin{gathered}
B_{\mathrm{m}}=\frac{U_{\mathrm{d} 1}}{2 N_{\mathrm{p}} f A_{\mathrm{cv}}}\left[\frac{1}{2}-\frac{L_{\sigma \mathrm{w}}}{L_{\mathrm{int}}} D_{\mathrm{s}}\right] \\
B_{\mathrm{ma}}=\frac{U_{\mathrm{d} 1}}{N_{\mathrm{p}} f A_{\mathrm{acv}}} \frac{L_{\sigma \mathrm{a}}}{L_{\mathrm{int}}} D_{\mathrm{s}} \\
\left|\frac{\mathrm{d} B_{\mathrm{m}}}{\mathrm{d} t}\right|=\left\{\begin{array}{l}
\frac{L_{\sigma \mathrm{a}}}{L_{\mathrm{int}}} \frac{U_{\mathrm{d} 1}}{N_{\mathrm{p}} A_{\mathrm{cv}}},\left[0, D_{\mathrm{s}} T\right] \\
\frac{U_{\mathrm{p}}}{N_{\mathrm{p}} A_{\mathrm{cv}}},\left[D_{\mathrm{s}} T, 0.5 T\right] \\
\frac{L_{\sigma a}}{L_{\mathrm{int}}} \frac{U_{\mathrm{d} 1}}{N_{\mathrm{p}} A_{\mathrm{cv}}},\left[0.5 T,\left(0.5+D_{\mathrm{s}}\right) T\right] \\
\frac{U_{\mathrm{d} 1}}{N_{\mathrm{p}} A_{\mathrm{cv}}},\left[\left(0.5+D_{\mathrm{s}}\right) T, T\right]
\end{array}\right. \\
\left|\frac{\mathrm{d} B_{\mathrm{ma}}}{\mathrm{d} t}\right|=\left\{\begin{array}{l}
\frac{2 L_{\sigma \mathrm{a}}}{L_{\mathrm{int}}} \frac{U_{\mathrm{d} 1}}{N_{\mathrm{p}} A_{\mathrm{acv}}},\left[0, D_{\mathrm{s}} T\right] \\
0,\left[D_{\mathrm{s}} T, 0.5 T\right] \\
\frac{2 L_{\sigma \mathrm{a}}}{L_{\mathrm{int}}} \frac{U_{\mathrm{d} 1}}{N_{\mathrm{p}} A_{\mathrm{acv}}},\left[0.5 T,\left(0.5+D_{\mathrm{s}}\right) T\right] \\
0,\left[\left(0.5+D_{\mathrm{s}}\right) T, T\right]
\end{array}\right.
\end{gathered}
$$

Based on the iGSE model [20], the engineering calculation model for the core losses under DAB excitation $\left(D_{\mathrm{h}}=0.5\right)$ is given as

$$
\begin{gathered}
P_{\mathrm{c}}=M_{\mathrm{c}} k_{\mathrm{i}} f^{\alpha-\beta}\left(\frac{U_{\mathrm{d} 1}}{N_{\mathrm{p}} A_{\mathrm{cv}}}\right)^{\beta}\left(\frac{1}{2}-\frac{L_{\sigma \mathrm{w}} D_{\mathrm{s}}}{L_{\mathrm{int}}}\right)^{\beta-\alpha}\left[1+2 D_{\mathrm{s}}\left(\frac{L_{\sigma \mathrm{a}}^{\alpha}}{L_{\mathrm{int}}^{\alpha}}-1\right)\right] \\
P_{\mathrm{ca}}=M_{\mathrm{ac}} k_{\mathrm{i}} 2^{\beta+1} f^{\alpha-\beta}\left(\frac{U_{\mathrm{d} 1}}{N_{\mathrm{p}} A_{\mathrm{acv}}} \frac{L_{\sigma \mathrm{a}}}{L_{\mathrm{int}}}\right)^{\beta} D_{\mathrm{s}}^{1-\alpha+\beta} \\
k_{\mathrm{i}}=\frac{k}{(2 \pi)^{\alpha-1} \int_{0}^{2 \pi}|\cos \theta|^{\alpha} 2^{\beta-\alpha} d \theta}
\end{gathered}
$$

where $P_{\mathrm{c}}$ is the loss of the main core; $P_{\mathrm{ca}}$ is the loss of the additional core; $M_{\mathrm{c}}$ is the effective weight of the main core; $M_{\mathrm{ac}}$ is the effective weight of the additional core; $f$ is frequency, $k_{\mathrm{i}}$ is the iGSE coefficient. $k, \alpha$, and $\beta$ are Steinmetz coefficients, which are extracted from sinusoidal measurement data of a gapped nanocrystalline core sample, which is shown in Figure 6.

The experiment is carried out with the core sample, and the calculated value and the measured value are compared in Figure 6, verifying the effectiveness of the model. 


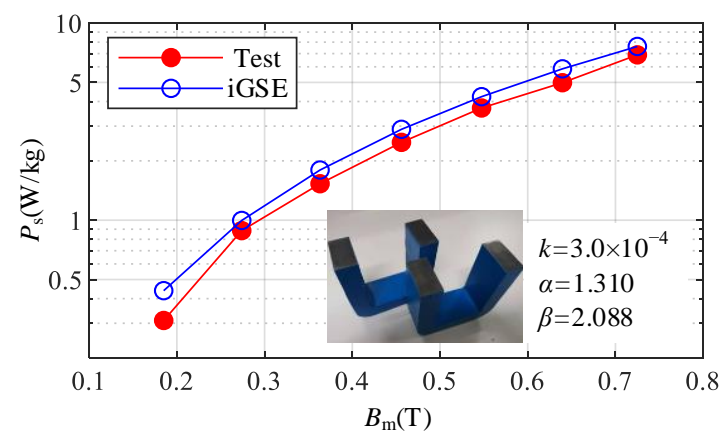

Figure 6. Comparison of experimental and calculated losses of the main core $\left(D_{\mathrm{h}}=0.5, D_{\mathrm{s}}=0\right)$.

\subsection{Winding Loss}

In order to calculate the winding losses under non-sinusoidal excitation, the current is usually decomposed by Fourier transform, and the winding loss is given as

$$
P_{\mathrm{Cu}}=\sum_{i=1}^{\infty} I_{\mathrm{RMS}-i}^{2} R_{\mathrm{AC}-i}=\sum_{i=1}^{\infty} I_{\mathrm{RMS}-i}^{2} F_{\mathrm{R}-i} R_{\mathrm{DC}}
$$

where $I_{\text {RMS- } i}$ is the $i_{\text {th }}$ harmonic components of the load current; the ac resistance coefficient $F_{\mathrm{R}}$ can be obtained by the Dowell model [21] as

$$
\begin{gathered}
F_{\mathrm{R}}=\Delta\left[\frac{\sinh 2 \Delta+\sin 2 \Delta}{\cosh 2 \Delta-\cos 2 \Delta}+\frac{2\left(m^{2}-1\right)}{3} \frac{\sinh \Delta-\sin \Delta}{\cosh \Delta+\cos \Delta}\right] \\
\Delta=d_{\mathrm{c}} \sqrt{\pi f \sigma \mu_{0} \frac{n h_{\mathrm{c}}}{h_{\mathrm{wd}}}}
\end{gathered}
$$

where, $m$ is the number of winding layers, $d_{c}$ is the thickness of conductor, $h_{\mathrm{c}}$ is the height of single conductor, $\Delta$ is the ratio of conductor thickness to penetrated depth; $\sigma$ is conductivity, $f$ is frequency, $\mu_{0}$ is the vacuum permeability, $n$ is the number of conductors per layer, and $h_{\mathrm{wd}}$ is the window height.

\subsection{Leakage Inductance of CW ELII MFT}

Figure 7 shows the simplified magnetic field distribution of CW ELII MFT. The integrated leakage inductance of CW ELII MFT consists of additional leakage inductance $L_{\sigma a}$, primary leakage inductance $L_{\sigma p}$, and secondary leakage inductance $L_{\sigma s}$. Since the leakage inductance of the primary winding and secondary winding are difficult to be separated physically, the two are usually combined as the winding leakage inductance $L_{\sigma \mathrm{\sigma}}$, so the total leakage inductance of the transformer is given as

$$
L_{\sigma}=L_{\sigma \mathrm{a}}+L_{\sigma \mathrm{p}}+L_{\sigma \mathrm{s}}^{\prime}=L_{\sigma \mathrm{a}}+L_{\sigma \mathrm{w}}
$$

The Rogowski model and Dowell model are classical calculation methods for transformer leakage inductance. The Rogowski model is a 2D static model in which the internal characteristics of the winding (for example, conductor thickness and inter-turn distance) and the effect of eddy current in the conductor are ignored. The Dowell model is a 1D dynamic model, and describes the influence of the eddy current on the leakage inductance based on the assumption of parallel flux and uniform arrangement of conductors [21]. The Dowell model is suitable for cases with eddy current effects but has an obvious error in cases with low utilization of the core window height [22]. Researchers introduced Rogowski coefficient into the Dowell model, making up for the deficiency of Dowell model [22,23]. The hybrid model is given as [22] 


$$
\begin{gathered}
L_{\text {ow }-\mathrm{H}}=\frac{N^{2} \mu_{0} l_{\mathrm{w}}}{h_{\mathrm{eq}}}\left[\frac{d_{\mathrm{cs}} m_{\mathrm{s}}}{3} F_{\mathrm{sw}}+\frac{d_{\mathrm{cp}} m_{\mathrm{p}}}{3} F_{\mathrm{pw}}+d_{\mathrm{d}}+\frac{d_{\mathrm{is}}\left(m_{\mathrm{s}}-1\right)\left(2 m_{\mathrm{s}}-1\right)}{6 m_{\mathrm{s}}}+\frac{d_{\mathrm{ip}}\left(m_{\mathrm{p}}-1\right)\left(2 m_{\mathrm{p}}-1\right)}{6 m_{\mathrm{p}}}\right] \\
F_{\mathrm{w}}=\frac{\left[\left(4 m^{2}-1\right) \frac{\sin h 2 \Delta-\sin 2 \Delta}{\cosh 2 \Delta-\cos 2 \Delta}-2\left(m^{2}-1\right) \frac{\sinh \Delta-\sin \Delta}{\cosh \Delta-\cos \Delta}\right]}{2 m^{2} \Delta} \\
\Delta=d_{\mathrm{c}} \sqrt{\mu_{0} \pi f \sigma \frac{n h_{\mathrm{c}}}{h_{\mathrm{wd}}}} \\
h_{\mathrm{eq}}=\frac{h_{\mathrm{w}}}{K_{\mathrm{R}}} \\
K_{\mathrm{R}}=1-\frac{1-\mathrm{e}^{-\pi h_{\mathrm{w}} / d_{\mathrm{w}}}}{\pi h_{\mathrm{w}} / d_{\mathrm{w}}}
\end{gathered}
$$

where $N$ is the turns of the winding; $l_{\mathrm{w}}$ is the average length of turns; $m_{\mathrm{p}}$ and $m_{\mathrm{s}}$ are respectively the numbers of primary and secondary winding layers; $\Delta$ is the ratio of conductor thickness to skin depth; $\sigma$ is conductivity; $f$ is frequency; $\mu_{0}$ is the vacuum permeability; $n$ is the number of conductors per layer; $K_{\mathrm{R}}$ is Rogowski coefficient; and $h_{\mathrm{eq}}$ is the corrected magnetic circuit length. As shown in Figure $8, d_{\mathrm{d}}$ is the main insulation distance; $d_{\mathrm{wp}}, d_{\mathrm{ws}}$, and $d_{\mathrm{w}}$, are respectively the width of the primary, secondary, and the entire winding; $h_{\mathrm{w}}$ is the height of winding; $h_{\mathrm{wd}}$ is the window height; $d_{\mathrm{ip}}$ and $d_{\mathrm{is}}$ are inter-layer distances; $d_{\mathrm{cp}}$ and $d_{\mathrm{cs}}$ are the widths of single conductors; $h_{\mathrm{cp}}$ or $h_{\mathrm{cs}}$ are the heights of conductors.

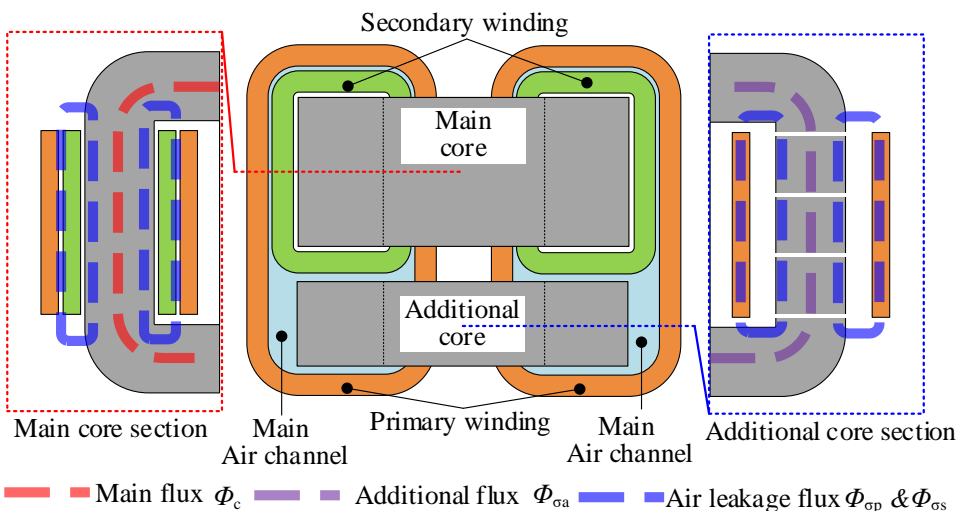

Figure 7. Simplified magnetic field distribution of CW ELII MFT.

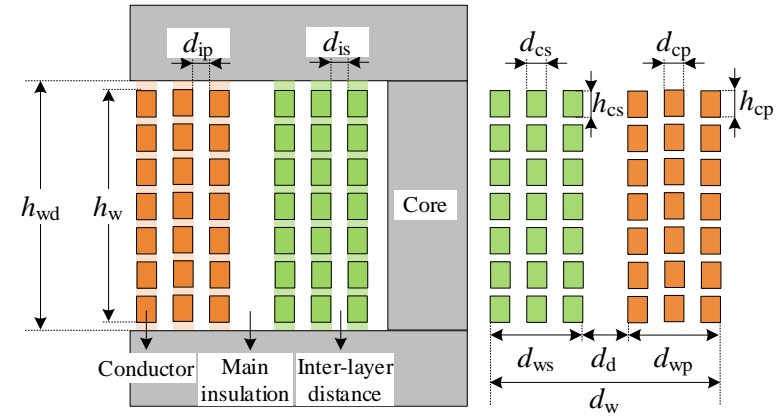

Figure 8. Winding parameters.

According to the assumption of the Dowell model, the main insulation layer has the same width $d_{\mathrm{d}}$ in the perimeter direction; conductor layers are arranged uniformly with the same 
inter-layer distance, i.e., $d_{\mathrm{is}}$ and $d_{\mathrm{ip}}$. As shown in Figure 7, the width of the main insulation in the CW ELII MFT is not equal in the circumference direction. In addition, when there is an internal air channel in the winding, the inter-layer distance will not be equal. Therefore, the original model needs to be improved to enable it to be used in the CW ELII MFT.

Assuming that the cross-sectional area of the main insulation layer is $S_{\mathrm{d}}$, the inductance of the main insulation layer can be obtained based on the magnetic field energy

$$
L_{\mathrm{d}}=\frac{N^{2} S_{\mathrm{d}} \mu_{0}}{h_{\mathrm{eq}}}
$$

Assuming that conductor layers are arranged non-uniformly with the inter-layer distances $d_{\mathrm{i}-1}, d_{\mathrm{i}-2}, \ldots, d_{\mathrm{i}-q}, \ldots, d_{\mathrm{i}-(m-1)}$, then the inter-layer inductance is given as

$$
L_{\mathrm{i}}=\frac{\mu_{0} N^{2} l_{\mathrm{w}}}{h_{\mathrm{eq}} m_{\mathrm{p}}^{2}} \sum_{q=1}^{q=m-1} q^{2} d_{\mathrm{i}-q}
$$

Replace the static terms in (19) by (24) and (25), and the mean length of turns of the primary and secondary windings, $l_{\mathrm{wp}}$ and $l_{\mathrm{ws}}$, are used to calculate the leakage inductance. The improved hybrid model is given as

$$
L_{\sigma \mathrm{w}-\mathrm{HI}}=\frac{N^{2} \mu_{0}}{h_{\mathrm{eq}}}\left[\frac{l_{\mathrm{wp}} d_{\mathrm{cp}} m_{\mathrm{p}}}{3} F_{\mathrm{wp}}+\frac{l_{\mathrm{ws}} d_{\mathrm{cs}} m_{\mathrm{s}}}{3} F_{\mathrm{ws}}+S_{\mathrm{d}}+\frac{l_{\mathrm{wp}}}{m_{\mathrm{p}}^{2}} \sum_{q=1}^{q=m_{\mathrm{p}}-1} q^{2} d_{\mathrm{ip}-q}+\frac{l_{\mathrm{ws}}}{m_{\mathrm{s}}^{2}} \sum_{r=1}^{r=m_{\mathrm{s}}-1} r^{2} d_{\mathrm{is}-r}\right]
$$

where, $d_{\mathrm{ip}-q}$ and $d_{\mathrm{is}}{ }^{-}$refer to the width of the $q_{\mathrm{th}}$ and $r_{\mathrm{th}}$ inter-layer distance of the primary and secondary winding from the outside to the inside.

A winding model with a non-uniform arrangement of conductors is shown in Figure 9. The model parameters are: 18 turns of the primary winding, 11 turns of the secondary, the width of the main insulating layer is $8 \mathrm{~mm}$, the thickness of the copper foil is $1 \mathrm{~mm}$, the height is $220 \mathrm{~mm}$, the height of the window is $245 \mathrm{~mm}$. An $8 \mathrm{~mm}$-wide air channel is located between the $q$ layer and the $q+1$ layer in the primary, and the other turns of the insulating layer have a width of $0.2 \mathrm{~mm}$.

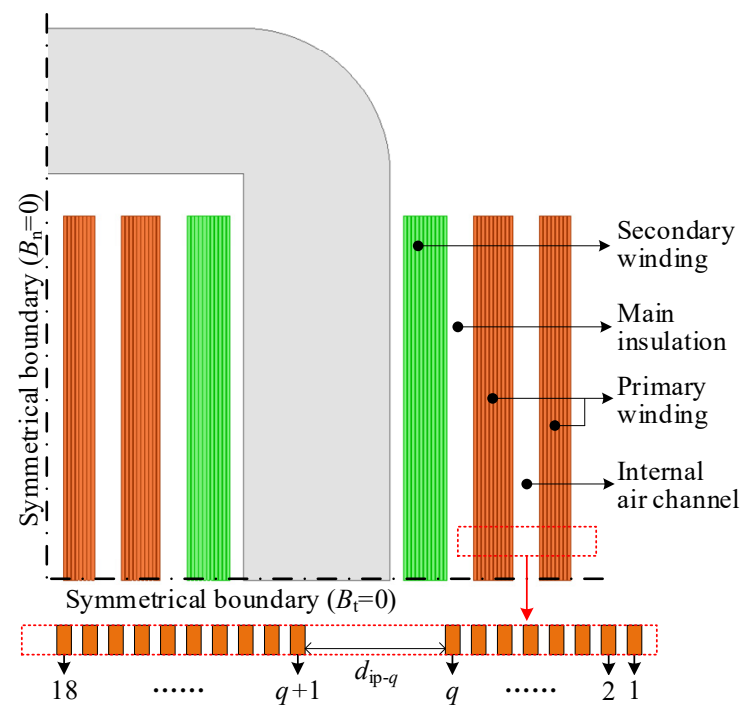

Figure 9. Winding model with non-uniform arrangement of conductor layers.

As shown in Figure 10, different models and FEM are used to calculate the leakage inductance of the example winding with different air channel locations. In the original and hybrid models, the average inter-layer width is used for the calculation. Both the FEM 
results and the calculation results of the proposed model increase as the position of the channel gradually approaches the main insulating layer, while the original model and the hybrid model cannot reflect the influence of the air channel on the leakage inductance. Based on the FEM results, the average error of the proposed model is $1.9 \%$, and the maximum error is $2.4 \%$, both smaller than the other two models; when $q=10-12$, the errors of the three models are relatively close, all less than $5 \%$.

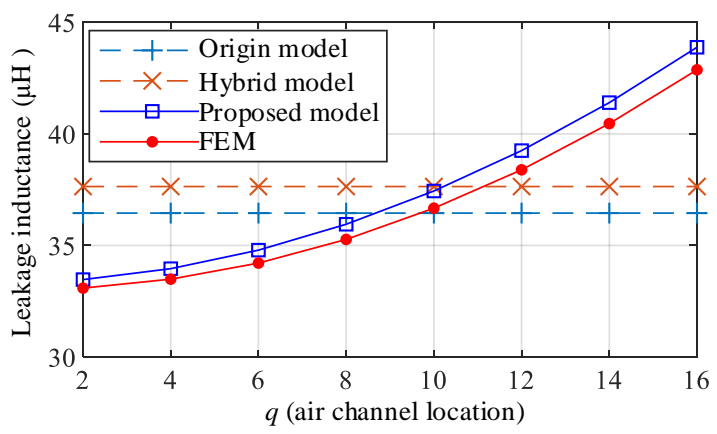

(a)

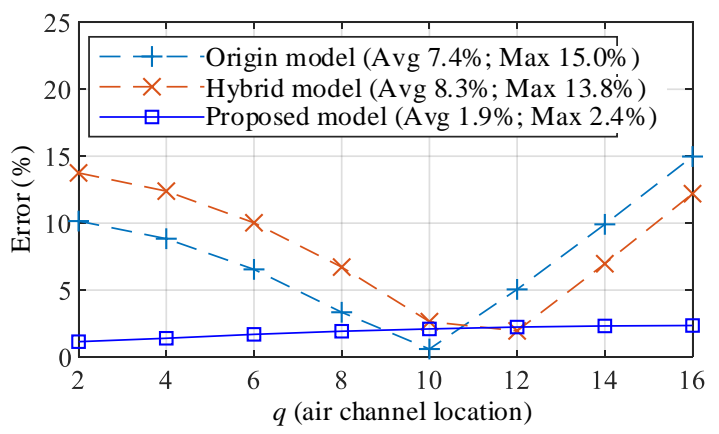

(b)

Figure 10. Comparison of FEM and analytical calculation results. (a) Comparison of results, (b) Comparison of errors.

The additional leakage inductance is composed of multiple air-gap inductances. In [24], an empirical formula is provided for calculating the inductance of the lumped air-gap inductor. In [25], the 2D expressions of air-gap reluctance are derived based on the SchwarzChristoffel transformation.

A simplified air gap is shown in Figure 11, and its reluctance is composed of the internal reluctance $R_{\mathrm{in}}$ and the fringing reluctance $R_{\mathrm{fr}}$ in parallel. The expressions are given as [26]

$$
\begin{gathered}
R_{\mathrm{in}}=\frac{d_{\mathrm{g}}}{\mu_{0} A_{\mathrm{ac}}} \\
R_{\mathrm{fr}}=\frac{\pi}{\mu_{0} C \ln \left(\frac{2 h+d_{\mathrm{g}}}{d_{\mathrm{g}}}\right)} \\
R_{\mathrm{g}-i}=R_{\mathrm{fr}}|| R_{\mathrm{in}}
\end{gathered}
$$

where $d_{\mathrm{i}}$ is the height of the air gap, $A_{\mathrm{ac}}$ is the cross-sectional area of the core, $\mu_{0}$ is air permeability, and $C$ is the perimeter of the cross-section.

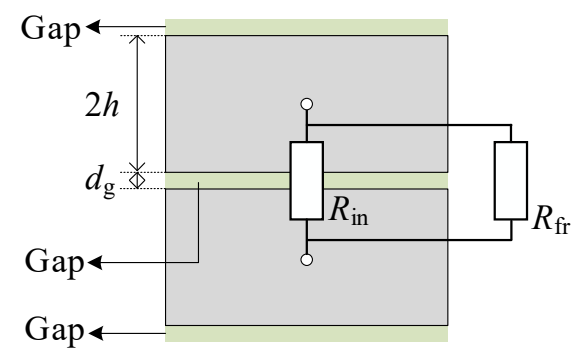

Figure 11. Simplified model of the air gap.

Thus, the additional leakage inductance is given as

$$
L_{\sigma \mathrm{a}}=\frac{N^{2}}{\sum R_{\mathrm{g}-i}}
$$

where $N$ is the number of turns of the winding. 
The FEM simulation is carried out to verify the calculation method for the additional leakage inductance, and the model and parameters are shown in Figure 12a. As shown in Figure 12b, as the air gap height increases, the inductance value is greatly reduced. Therefore, in the MFT design process, the height and number of the air gap are used to adjust the inductance value.

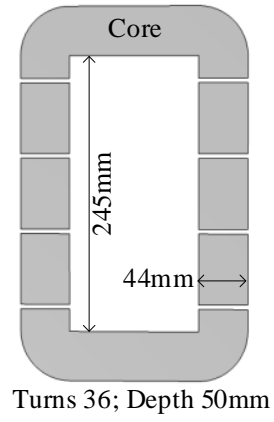

(a)

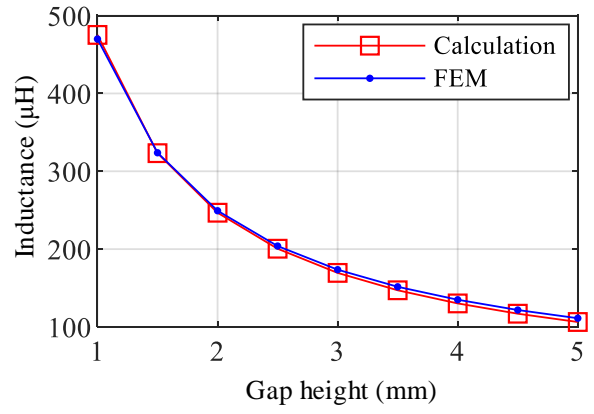

(b)

Figure 12. Calculation and simulation of the additional leakage inductance. (a) FEM model (b) comparison of the calculated and simulated values.

\section{Thermal Modeling and Analysis}

For the design of the air-water combined cooling MFT, a thermal model is necessary. The steady-state thermal model mainly includes the heat source, thermal resistance, and network topology. The heat source, namely the core and winding losses, has been discussed above. Therefore, this section mainly discusses the modeling of thermal resistance and network topology.

\subsection{Convective Thermal Resistance of the Air Channel}

The air channel is an important heat dissipation structure of transformer windings. The air channel can be simplified as a parallel-plate channel for thermal modeling. As shown in Figure 13, the length of the air channel is $L$, the cross-section is $W \times S$, and $W>S$. The hydraulic diameter is given as [27]

$$
d_{\mathrm{h}}=\frac{4 A}{P}=\frac{4 W S}{2(W+S)} \approx 2 S
$$

where $A$ is the cross-section area and $P$ is the inner perimeter.

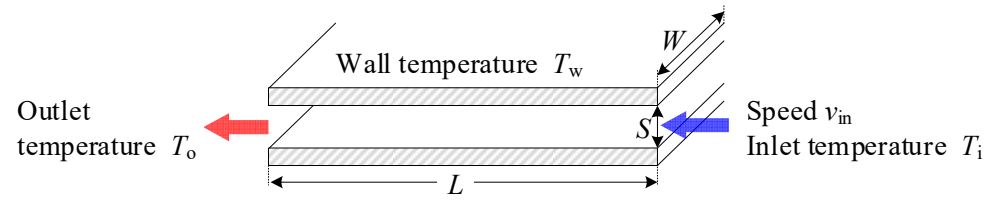

Figure 13. Parallel-plate air channel.

Since the convection model of the parallel-plate channel in literature is not complete, a combination of the laminar-flow model [28] for the parallel-plate channel and a turbulentflow model [28] for the non-circular tube is adopted in this paper. At the same time, linear interpolation is used in the transition period between laminar flow and turbulent flow. The Nusselt number calculation formula of the parallel-plate air channel is given as

$$
N_{\mathrm{u}}=\left\{\begin{array}{l}
{\left[N_{1}^{3}+N_{2}{ }^{3}+N_{3}^{3}\right]^{1 / 3},\left(R_{\mathrm{e}} \leq 2300\right)} \\
\frac{\left(\frac{f_{\mathrm{r}}}{8}\right) R_{\mathrm{e}} P_{\mathrm{r}}\left[1+\left(\frac{d_{\mathrm{h}}}{L}\right)^{2 / 3}\right]\left(\frac{T_{\mathrm{f}}}{T_{\mathrm{w}}}\right)^{0.45}}{1+12.7 \sqrt{\frac{f_{\mathrm{r}}}{8}}\left(P_{\mathrm{r}}^{2 / 3}-1\right)},\left(R_{\mathrm{e}} \geq 10^{4}\right) \\
(1-r) N_{\mathrm{u} 2300}+r N_{\mathrm{u} 10000},\left(2300<R_{\mathrm{e}}<10^{4}\right)
\end{array}\right.
$$




$$
\begin{gathered}
R_{\mathrm{e}}=\frac{\rho v_{\mathrm{in}} d_{\mathrm{h}}}{\eta} \\
N_{1}=7.541 \\
N_{2}=1.841\left(R_{\mathrm{e}} P_{\mathrm{r}} d_{\mathrm{h}} / L\right)^{1 / 3} \\
N_{3}=\left(\frac{2}{1+22 P_{\mathrm{r}}}\right)^{1 / 6}\left(R_{\mathrm{e}} P_{\mathrm{r}} d_{\mathrm{h}} / L\right)^{1 / 2} \\
f_{\mathrm{r}}=\left(1.8 \log _{10} R_{\mathrm{e}}-1.5\right)^{-2} \\
P_{\mathrm{r}}=\frac{c_{\mathrm{p}} \eta}{\lambda_{\mathrm{f}}} \\
T_{\mathrm{f}}=\frac{T_{\mathrm{i}}+T_{\mathrm{o}}}{2} \\
r=\frac{R_{\mathrm{e}}-2300}{10^{4}-2300}
\end{gathered}
$$

where $R_{\mathrm{e}}$ is the Reynolds number; $N_{1}, N_{2}$, and $N_{3}$ are the laminar flow model coefficients; $P_{\mathrm{r}}$ is the Prandtl number; $T_{\mathrm{f}}$ is the average fluid temperature; $T_{\mathrm{i}}$ is the inlet temperature, which is equal to the ambient environment temperature $T_{\mathrm{am}} ; T_{\mathrm{o}}$ is the outlet temperature; $T_{\mathrm{W}}$ is the wall temperature; $f_{\mathrm{r}}$ is the friction coefficient; $r$ is the interpolation coefficient in the transition period; $\rho$ is the fluid density; $\eta$ is dynamic viscosity; $c_{\mathrm{p}}$ is fluid specific heat capacity; and $\lambda_{\mathrm{f}}$ is fluid thermal conductivity.

After the Nusselt number is obtained, the surface heat transfer coefficient is given as

$$
h=\frac{\lambda_{\mathrm{f}} N_{\mathrm{u}}}{d_{\mathrm{h}}}
$$

The FEM simulation is carried out to verify the accuracy of the above calculation methods. Figure 14 shows the temperature distribution inside the air channel under different wind velocities. The simulated and calculated results are compared in Figure 15. As shown in Figure 15a, the average heat transfer coefficient increases with the higher wind velocity. The reason is that the higher wind velocity reduces the thickness of the boundary layer. As shown in Figure 15b, the shorter channel can achieve higher average heat transfer coefficients. The reason is that the thickness of the boundary layer increases with the channel length. The error between the calculated and simulated result is less than $10 \%$.

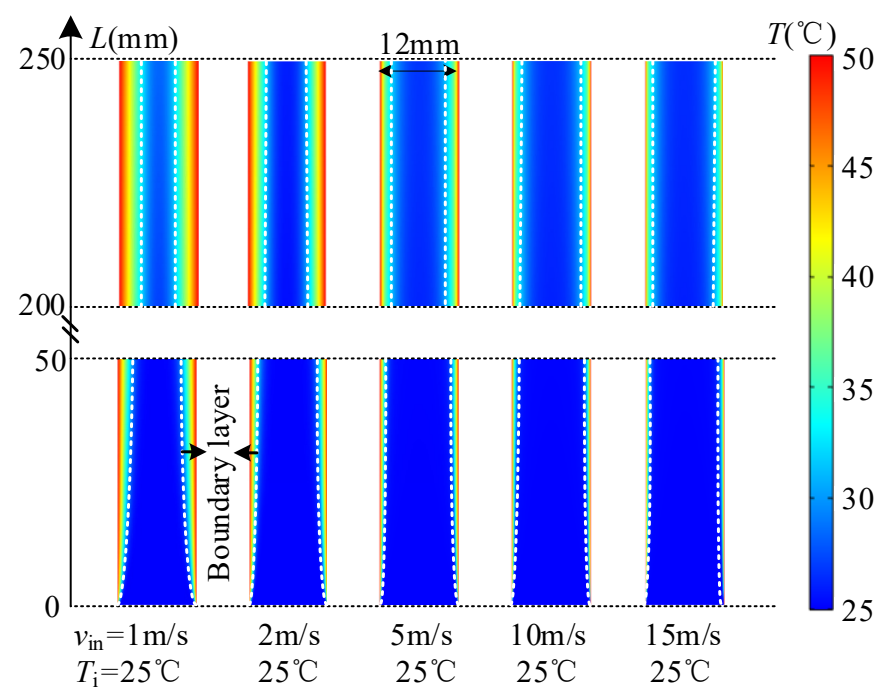

Figure 14. Temperature distribution of the air channel under different wind velocities. 


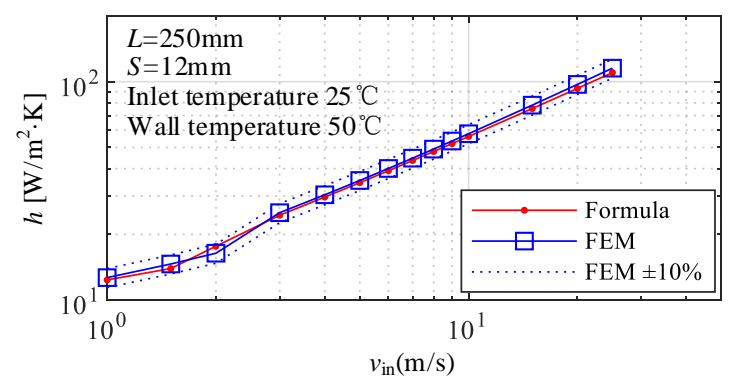

(a)

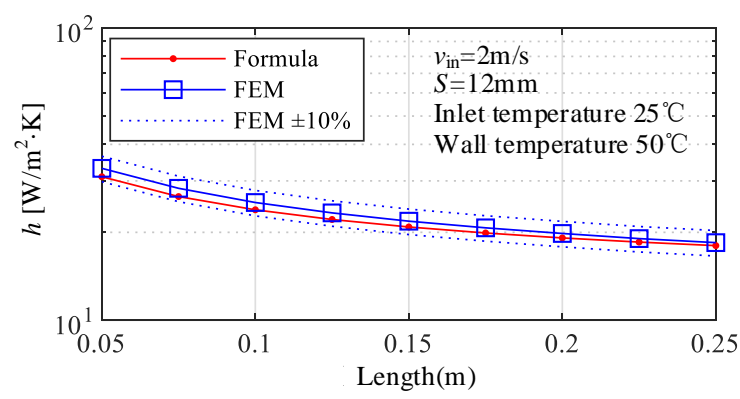

(b)

Figure 15. Comparison of the simulated and calculated heat transfer coefficients. (a) Different wind velocities; (b) different channel lengths.

After obtaining the heat transfer coefficient, the convective thermal resistance can be obtained according to the Newton cooling formula of the internal convection.

$$
Q=h A\left(T_{\mathrm{w}}-\frac{T_{\mathrm{i}}+T_{\mathrm{o}}}{2}\right)=\frac{\left(T_{\mathrm{w}}-T_{\mathrm{f}}\right)}{R_{\text {inconv }}^{\prime}}
$$

In (42), the temperature difference is between the surface temperature $T_{\mathrm{w}}$ and the fluid temperature $T_{\mathrm{f}}$. As the fluid temperature $T_{\mathrm{f}}$ is related to the internal convection process, the thermal resistance $R_{\text {inconv }}$ defined by (42) cannot be directly connected to the external environment nodes in the thermal network. Therefore, transform (42) as

$$
Q=\frac{h A\left(T_{\mathrm{w}}-\frac{T_{\mathrm{i}}+T_{\mathrm{o}}}{2}\right)}{\left(T_{\mathrm{w}}-T_{\mathrm{i}}\right)}\left(T_{\mathrm{w}}-T_{\mathrm{i}}\right)=\frac{\left(T_{\mathrm{w}}-T_{\mathrm{i}}\right)}{R_{\text {inconv }}}
$$

The thermal resistance $R_{\text {inconv }}$ defined by (43) can be directly connected to the external environment nodes for the temperature difference is between surface temperature $T_{\mathrm{w}}$ and inlet temperature $T_{\mathrm{i}}$. The proposed thermal resistance definition for the internal convection is more convenient for thermal network modeling.

\subsection{Conduction and Radiation Thermal Resistance}

Conduction: for a uniform cuboid with length $l$, thermal conductivity $\lambda$, and crosssectional area $A$, the thermal resistance is given as [27]

$$
R_{\mathrm{cond}}=\frac{l}{\lambda A}
$$

Radiation: for a surface with the area $A_{\text {sur }}$, the surface temperature $T_{\text {sur, }}$ and the ambient temperature $T_{\mathrm{am}}$, the thermal radiation resistance is given as [27]

$$
R_{\mathrm{rad}}=\frac{T_{\text {sur }}-T_{\mathrm{am}}}{\varepsilon_{\mathrm{i}} \sigma A_{\mathrm{sur}}\left(T_{\mathrm{sur}}^{4}-T_{\mathrm{am}}^{4}\right)}
$$

where $\sigma$ is Boltzmann constant and $\varepsilon_{\mathrm{i}}$ is emissivity factor.

\subsection{Thermal Network Topology}

The structure of the air-water cooled CW ELII MFT is shown in Figure 16. As shown in Figure 16b, to reduce the eddy current loss caused by the fringing flux, the tooth-slot structure is adopted in water-cooling plates adjacent to the additional core. 


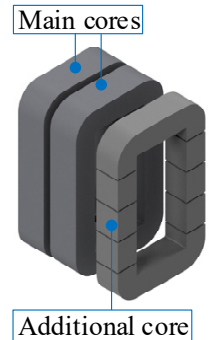

(a)

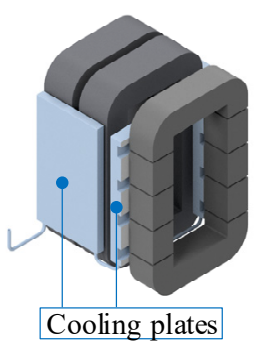

(b)

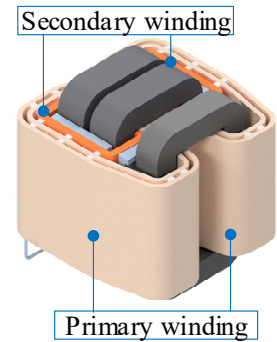

(c)

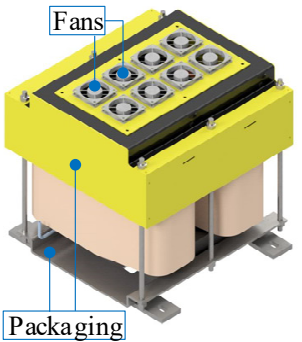

(d)

Figure 16. The structure of CW ELII MFT. (a) Cores; (b) water-cooling system; (c) windings; (d) packaging and fans.

As shown in Figure 17, the thermal network model has ten nodes. $N_{1} \sim N_{3}$ denote the upper yoke, the limb, and the lower yoke of the main core, respectively. $N_{4}$ represents the secondary winding. $N_{5}$ represents the primary winding. $N_{6} \sim N_{8}$ represent the upper yoke, the limb, and the lower yoke of the additional core. $N_{9}$ represents the external air. $N_{10}$ represents the water-cooling plate. The additional nodes $n_{1} \sim n_{8}$ represent the convection and radiation surfaces of nodes $N_{1} \sim N_{8}$ and are used to provide the wall temperature for convection and radiant thermal resistance calculations. $Q_{1}-Q_{8}$ represent the losses of each node, respectively; $T_{\mathrm{am}}$ and $T_{\mathrm{co}}$ represent the temperature of the environment and the water-cooling plate. In the above model, the internal forced convection process of the water-cooling plate is ignored. The implication of $R_{x y \text {-Cond/Conv/Rad }}$ is conduction/convection/radiation thermal resistance from node $x$ to node $y$. In a steady-state, the governing equation is given as [12]

$$
0=A T+B U
$$

where $\boldsymbol{A}$ describes the thermal resistance network of internal nodes; $\boldsymbol{B}$ describes the thermal resistance network between the internal nodes and the external nodes (air and water-cooling plates); $T$ is the node temperature vector; $U$ is the external excitation vector.

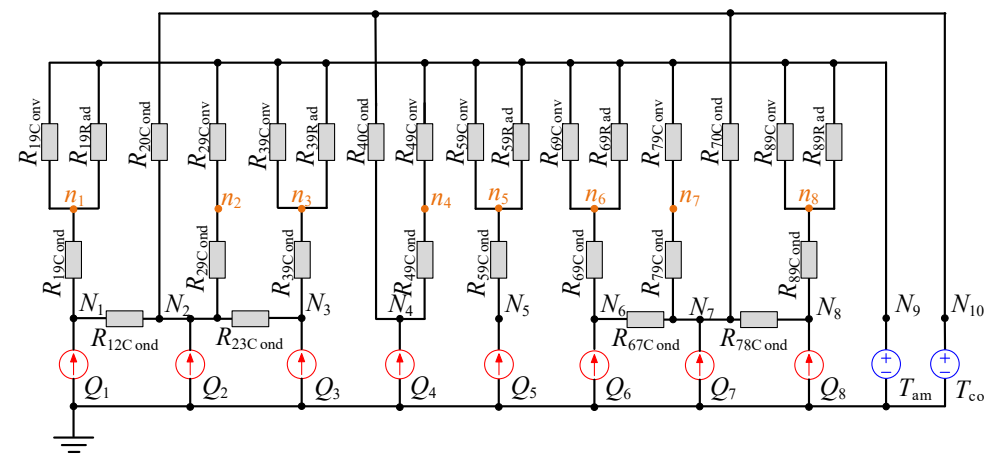

Figure 17. Thermal network model of the CW ELII MFT.

\section{Optimal Design, Analysis, and Example}

The MFT design is a non-linear, non-convex, and non-continuous problem. The optimization design and the comparative evaluation of the inductor-integrated MFT is presented in this section.

\subsection{Design Method}

The critical factors of optimal design include: design inputs, constraints, and optimization objectives. 


\subsubsection{Design Inputs}

As shown in Figure 18, there are many variables in the optimal design of MFT, and it is unrealistic to optimize all variables. Therefore, seven free variables are selected in this paper, including the flux density of main core $B_{\mathrm{m}}$, the flux density of additional core $B_{\mathrm{ma}}$, turns of the primary winding $N_{\mathrm{p}}$, the height of foils $h_{\mathrm{c}}$, the thickness of primary foils $d_{\mathrm{cp}}$, the thickness of secondary foils $d_{\mathrm{cs}}$, and the width of core tape $D_{\mathrm{cs}}$. The free variables constitute the input vector of the optimization design as

$$
\boldsymbol{x}=\left[B_{\mathrm{m}}, B_{\mathrm{ma}}, N_{\mathrm{p}}, h_{\mathrm{c}}, d_{\mathrm{cp}}, d_{\mathrm{cs}}, D_{\mathrm{cs}}\right]
$$
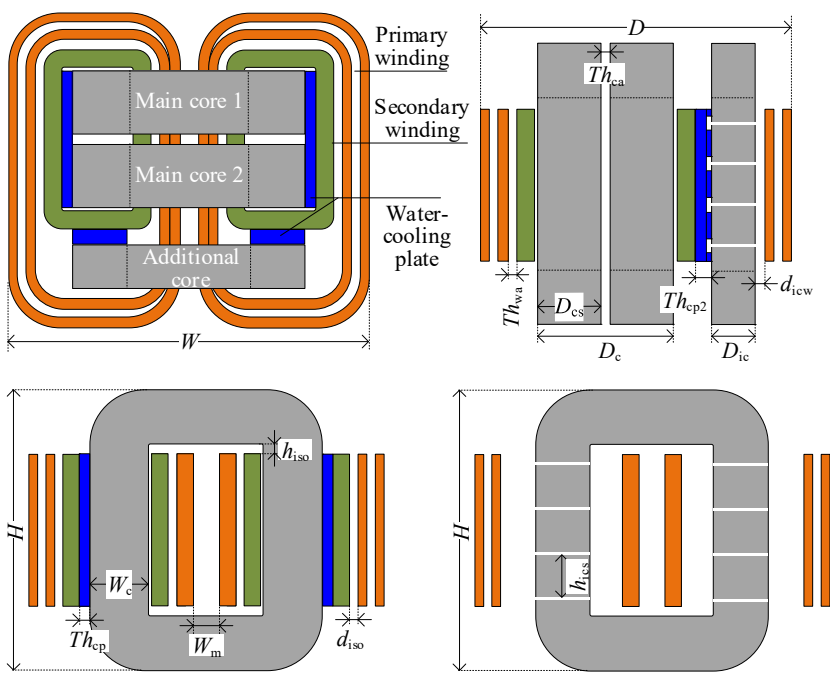

Figure 18. Sketch of the CW ELII MFT structure.

In addition, inputs design includes many fixed parameters about operating conditions and material properties, such as the DC voltage $U_{\mathrm{dc} 1}$ and $U_{\mathrm{dc} 2}$, the turns ratio of the transformer $k_{\mathrm{PS}}$, frequency $f$, and electrical and thermal properties of materials.

\subsubsection{Constraints}

The design constraints include temperature rise $\Delta T_{\max }$, leakage inductance error $e_{\mathrm{Lmax}}$, and the spatial size $H_{\max }, W_{\max }, D_{\max }$.

$$
\text { s.t. }\left\{\begin{array}{l}
\Delta T<\Delta T_{\max } \\
H<H_{\max } \\
W<W_{\max } \\
D<D_{\max } \\
\left|\frac{L_{\sigma}-L_{\sigma t}}{L_{\sigma t}}\right|<e_{L \max }
\end{array}\right.
$$

where $\Delta T$ is the temperature increase; $H, W$, and $D$ are spatial sizes; and $L_{\sigma t}$ is the target leakage inductance.

\subsubsection{Objectives}

In general, efficiency and power density are the main parameters for evaluating the design results.

$$
\begin{aligned}
& f_{1}(\boldsymbol{x})=\max \eta(\boldsymbol{x})=\max \left(\frac{P_{\text {out }}}{P_{\text {out }}+P_{\mathrm{Fe}}+P_{\mathrm{Cu}}}\right) \\
& f_{2}(\boldsymbol{x})=\max P_{d}(\boldsymbol{x})=\max \left(\frac{P_{\text {out }}}{V}\right)
\end{aligned}
$$

where $\eta$ is efficiency, $P_{\text {out }}$ is the output power, $P_{\mathrm{Fe}}$ is the core loss, $P_{\mathrm{Cu}}$ is the winding loss, $V$ is the volume of MFT (ignoring structural components and terminals). 
The design procedures of inductor-integrated MFT are shown in Figure 19. The brute force grid search method, which is extremely robust, is adopted on the premise that the calculation time is acceptable. According to the free parameters of the design input, a design space is constructed, which contains all possible designs. Designs in the space are calculated, and the results are saved. According to the constraints, the qualified designs are obtained. The Pareto front is solved according to the optimization objectives, and the designer can determine the final design according to the front.

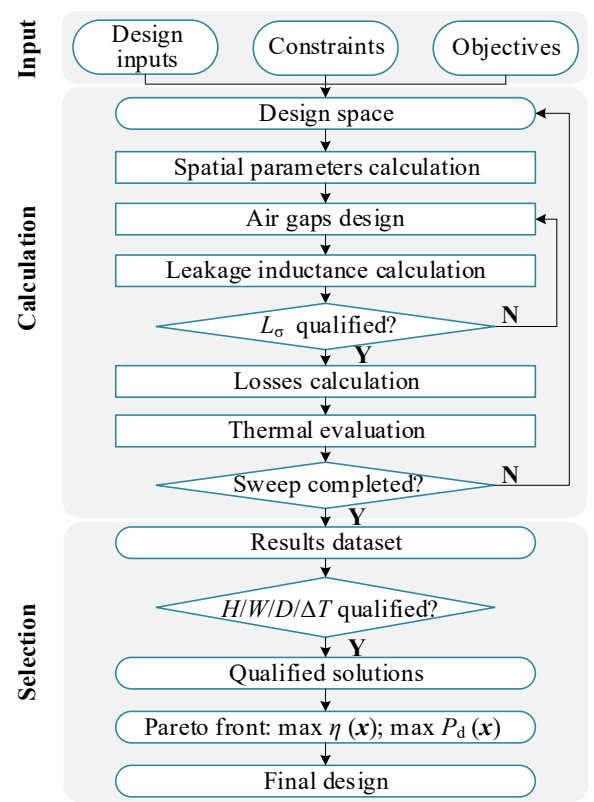

Figure 19. Design procedures of the CW ELII MFT.

\subsection{Design Results and Comparative Analysis}

The $4 \mathrm{kHz}, 200 \mathrm{~kW}, 200 \mu \mathrm{H}$ inductor-integrated MFT for a DAB converter is taken as an example of optimal design, and the main parameters and constraints are shown in Table 1.

Table 1. Parameters and constraints.

\begin{tabular}{ccc}
\hline Parameters & Description & Value \\
\hline$P$ & rated power & $200 \mathrm{~kW}$ \\
$U_{\mathrm{d} 1} / U_{\mathrm{d} 2}$ & MV / LV DC voltage & $1.6 \mathrm{kV} / 1 \mathrm{kV}$ \\
$k_{\mathrm{PS}}$ & turns ratio & 1.6 \\
$D_{\mathrm{h}}$ & H-bridge duty & 0.5 \\
$D_{\mathrm{s}}$ & phase-shifting duty & 0.0875 \\
$f$ & fundamental frequency & $4 \mathrm{kHz}$ \\
$L_{\sigma \mathrm{t}}$ & target leakage inductance & $200 \mu \mathrm{H}$ \\
$e_{\mathrm{Lmax}}$ & max leakage error & $5 \%$ \\
$H_{\max } \times W_{\max } \times D_{\max }$ & space limit & $0.4 \times 0.4 \times 0.4 \mathrm{~m}$
\end{tabular}

The optimal designs of the CW LII MFT and the CW ELII MFT are carried out, respectively. The CW LII MFT is also combined air-water cooling, the difference is that the CW LII structure has no additional core, and the target leakage inductance is achieved by adjusting the width of the main insulation layer. The SW LII structure and the SW ELII structure are not discussed in this paper due to the inevitable external leakage flux.

Figure 20 shows the comparison of qualified designs of the two integration structures. The designs with the maximum power density in the CW LII and the CW ELII qualified solutions are shown in Table 2, and only the transformer body and internal cooling plates are considered in the power density specification. The CW ELII design has a lower core 
loss, but the eddy current of the water-cooling plate makes the efficiency of the CW ELII solution $0.09 \%$ lower than that of the CW LII solution. The volume power density of the CW LII solution is $2.24 \mathrm{~kW} / \mathrm{dm}^{3}$, and that of the CW ELII solution is $5.16 \mathrm{~kW} / \mathrm{dm}^{3}$, which is about $130 \%$ higher. Meanwhile, the weight power density of the CW ELII solution is only 9.9\% higher than that of the CW LII solution, indicating that the main advantage of the CW ELII structure is the more compact structure. Considering the core material and wire material, the unit cost of the CW ELII design is $7.7 \%$ lower than that of the CW LII design.

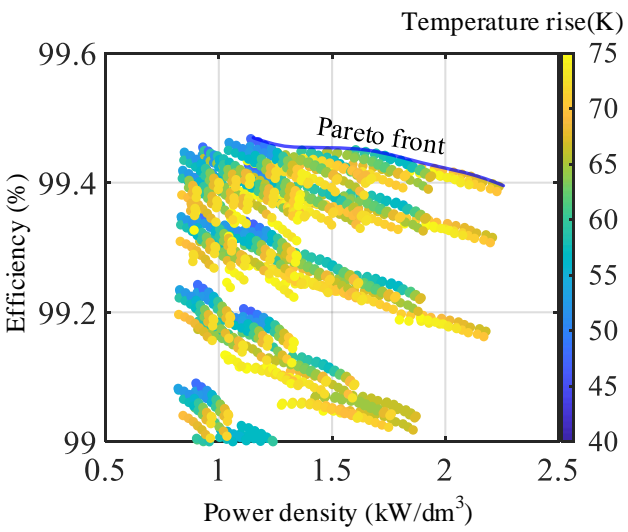

(a)

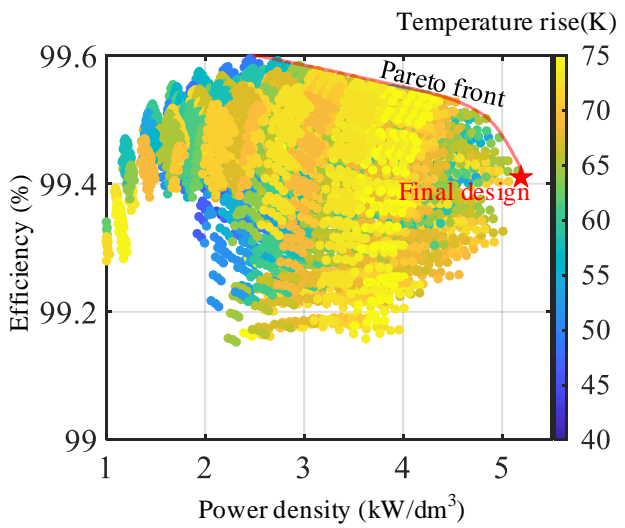

(b)

Figure 20. Comparison of CW LII and CW ELII qualified designs. (a) CW LII qualified designs; (b) CW ELII qualified designs.

Table 2. Comparison of CW LII and CW ELII solutions.

\begin{tabular}{|c|c|c|c|}
\hline \multicolumn{2}{|c|}{ Parameters } & CW LII & CW ELII \\
\hline \multicolumn{2}{|c|}{ Power $(\mathrm{kW})$} & 200 & 200 \\
\hline \multicolumn{2}{|c|}{ Leakage inductance $(\mu \mathrm{H})$} & 200 & 200 \\
\hline \multirow{4}{*}{ Winding } & Turns & $32 / 20$ & $36 / 22$ \\
\hline & Foil size $(\mathrm{mm})$ & $0.4 \times 200$ & $0.4 \times 200$ \\
\hline & Air channel width (mm) & 86.3 & 12 \\
\hline & Winding loss $(\mathrm{W})$ & 913.7 & 916.8 \\
\hline \multirow{2}{*}{ Main core } & Flux density $(\mathrm{T})$ & 0.65 & 0.65 \\
\hline & No-load core loss (W) & 305.4 & 200.9 \\
\hline \multirow{3}{*}{ Additional core } & Flux density $(\mathrm{T})$ & / & 0.4 \\
\hline & Load core loss $(\mathrm{W})$ & / & 56.8 \\
\hline & Cooling plate loss (W) & / & 244 \\
\hline \multicolumn{2}{|c|}{ Temperature rise $(\mathrm{K})$} & 66 & 64.1 \\
\hline \multicolumn{2}{|c|}{ Efficiency (\%) } & 99.39 & 99.30 \\
\hline \multicolumn{2}{|c|}{ Unit cost (RMB/kW) } & 78 & 72 \\
\hline \multicolumn{2}{|c|}{ Power density $(\mathrm{kW} / \mathrm{kg})$} & 2.63 & 2.88 \\
\hline \multicolumn{2}{|c|}{ Power density $\left(\mathrm{kW} / \mathrm{dm}^{3}\right)$} & 2.24 & 5.16 \\
\hline
\end{tabular}

In order to make a more comprehensive comparison of the two structures, the optimal designs for different leakage inductance values are carried out. It should be noted that other design inputs remain unchanged among the designs. The comparison of the Pareto fronts is shown in Figure 21. As shown in Figure 21a, when the leakage inductance is 10\% p.u. $(40 \mu \mathrm{H})$, the Pareto fronts of the CW LII solutions and the CW ELII solutions are very close. However, with the increase of leakage inductance, the power density of CW LII decreases continuously, while the CW ELII has not changed significantly. 


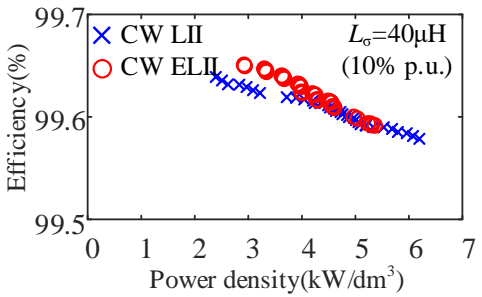

(a)

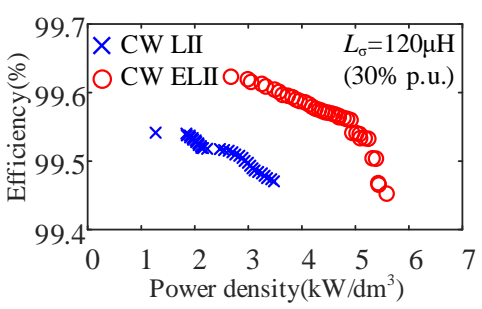

(c)

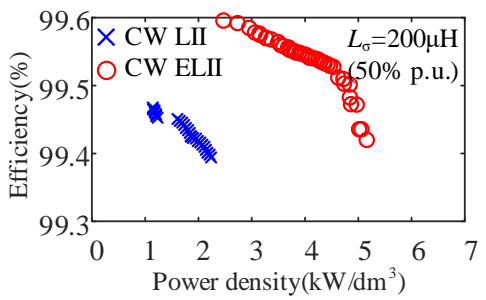

(e)

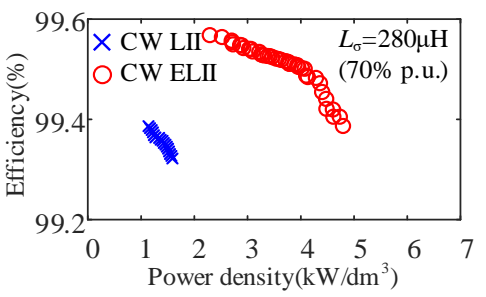

(g)

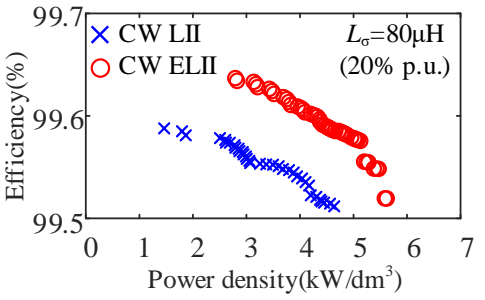

(b)

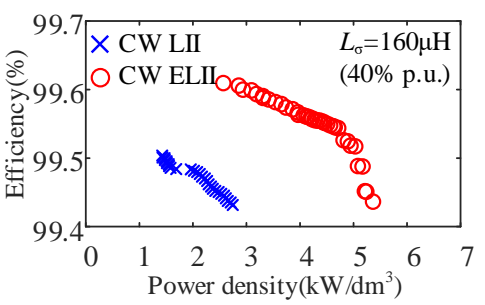

(d)

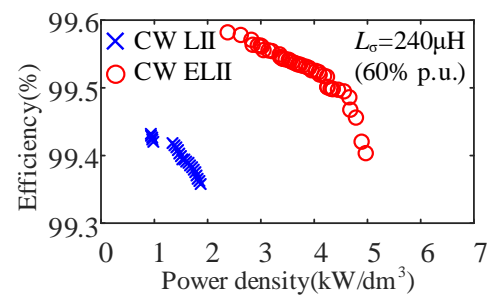

(f)

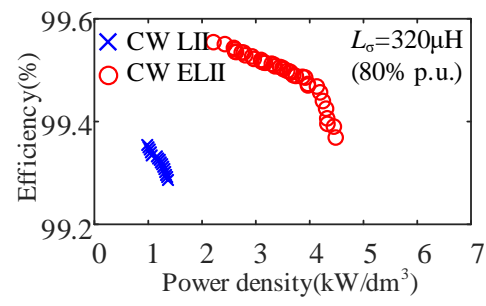

(h)

Figure 21. Pareto fronts of CW LII and CW ELII solutions with different leakage inductances. (a-h) leakage inductance: $10 \%$ p.u. to $80 \%$ p.u. $(40 \mu \mathrm{H}$ to $320 \mu \mathrm{H})$.

Based on Figure 21, the CW LII and CW ELII solutions with the highest power density in each group are selected for comparison, as shown in Figure 22. As shown in Figure 22a, the power density of the CW LII solution decreases with larger leakage inductance, and the maximum power density is $6.2 \mathrm{~kW} / \mathrm{dm}^{3}$ at $10 \%$ p.u. $(40 \mu \mathrm{H})$. The CW ELII solution achieves the maximum power density of $5.8 \mathrm{~kW} / \mathrm{dm}^{3}$ at $20 \%$ p.u. $(80 \mu \mathrm{H})$. When the leakage inductance per unit value exceeds $20 \%$ (p.u.), the CW ELII solution can achieve a higher power density than the CW LII solution. It becomes more evident as the leakage inductance increases. As shown in Figure 22b, as the leakage inductance increases, the efficiency of the two solutions is gradually reduced. When the leakage inductance per unit value is greater than $40 \%$ (p.u.), the CW ELII solution has advantages.

While keeping other parameters unchanged, the rated current in the design input is changed to analyze the influence of the power on the design result. In all groups, the leakage inductance in per-unit is 50\%. Figure 23 shows the comparison of the Pareto fronts of CW ELII MFT designs with different power requirements. As the power increases, it is easier to achieve higher power densities. A possible reason is that the insulation distance in the MFT is a fixed parameter under the same insulation requirement. A possible reason is that the insulation distance in the MFT is a fixed parameter under the same insulation requirement. 
With the increase of rated current $(80 \mathrm{~kW}$ to $280 \mathrm{~kW})$, the proportion of windings in the core window increases and the proportion of insulating space decreases, which makes the MFT power density increase. When the rated current is large enough (280 kW and $320 \mathrm{~kW})$, the proportion of insulating space is small, and this effect is no longer obvious.

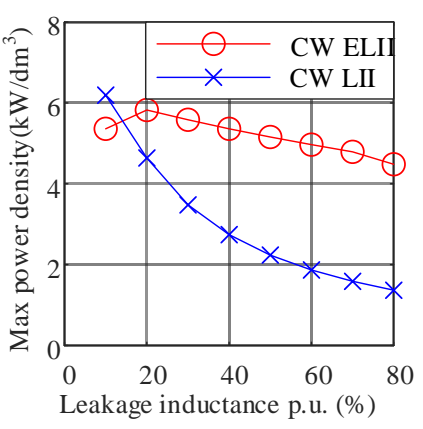

(a)

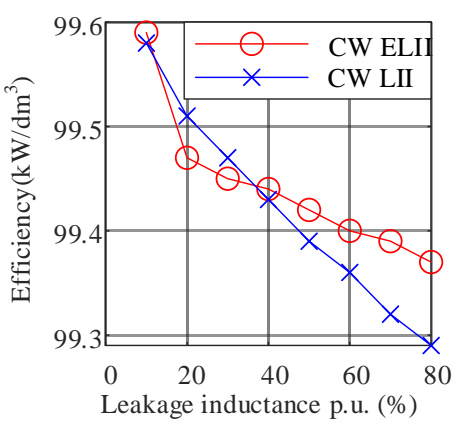

(b)

Figure 22. Comparison of solutions for different inductance requirements. (a) Max power density; (b) efficiencies.

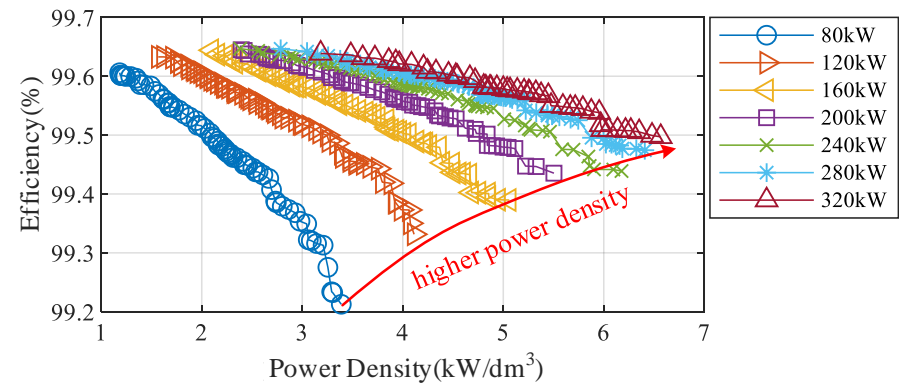

Figure 23. Comparison of Pareto fronts of CW ELII MFT designs under different power requirements (different current amplitudes; same leakage inductance in per unit, 50\%).

\subsection{Prototype and Experimental Verification}

According to the final design of the CW ELII MFT, a prototype was manufactured and tested in a $200 \mathrm{~kW}$ DAB converter, which is a module of a 2 MW MMC-BDC. The MFT prototype and the experimental platform are shown in Figure 24.

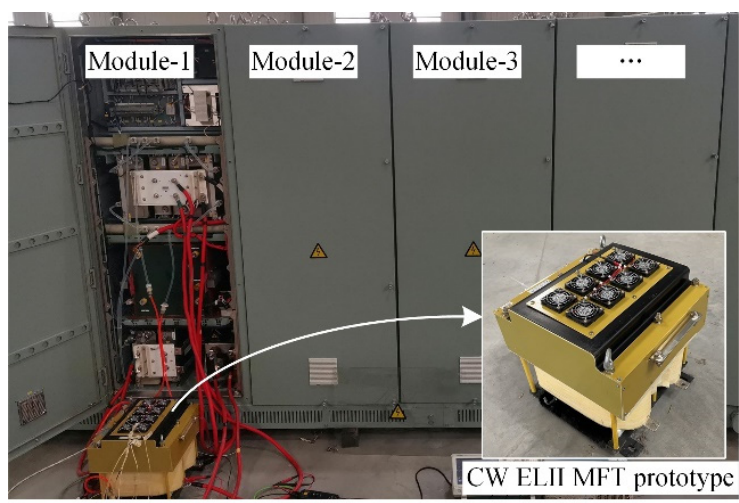

Figure 24. Experimental platform and the MFT prototype.

Figure 25 shows the waveforms of the no-load voltage and current under LV side excitation. The voltage was measured by Tek P5210A voltage probe (bandwidth $50 \mathrm{MHz}$ ), 
and the current was measured by Tek TCP303 + TCPA300 current probe (bandwidth $15 \mathrm{MHz}$ ). The no-load loss can be calculated by the no-load voltage and current.

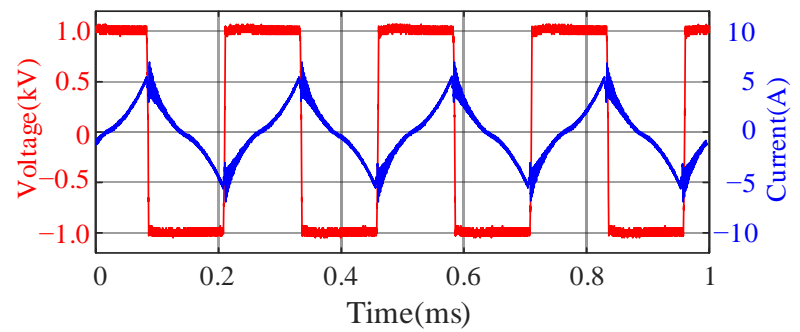

Figure 25. Voltage and current waveforms of the no-load condition.

Figure 26 shows the voltage and current waveforms under the load condition with an output power of $200 \mathrm{~kW}$, in which the DC voltages is $1.6 \mathrm{kV} / 1 \mathrm{kV}$ and the phase-shifting duty is 0.0875 . The temperature rise experiment was carried out under this condition, where the inlet water temperature was $35.5^{\circ} \mathrm{C}$, the ambient temperature was $39{ }^{\circ} \mathrm{C}$, and the average inlet wind speed was $1.0 \mathrm{~m} / \mathrm{s}$ (measured by KIMO VT110). Figure 27 shows the MFT surface temperature distribution in thermal steady state. Contact sensors were placed on the core surface to monitor the core temperature. At the same time, to avoid the influence of internal wind on winding temperature measurement, the resistance method was used to measure the average winding temperature.
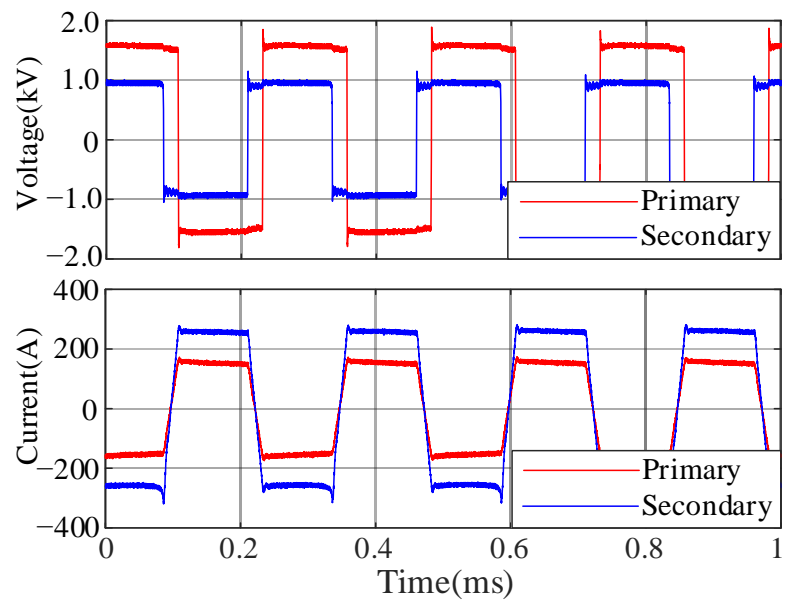

Figure 26. Voltage and current waveforms of the load condition.

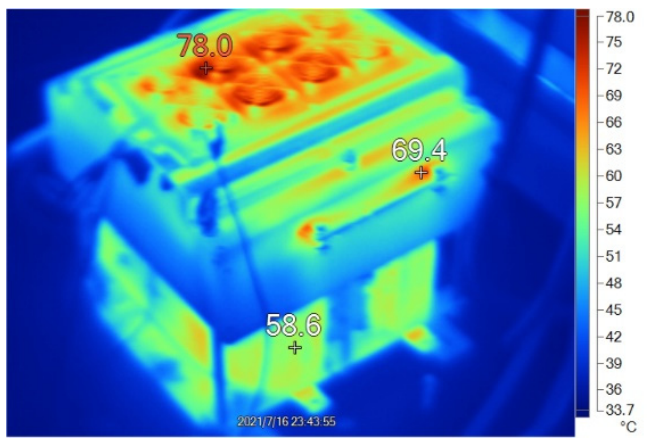

Figure 27. Temperature distribution measured by the infrared thermal camera.

The experimental results are summarized as in Table 3. The experimental results of the inductance, no-load loss, and temperature increase are basically consistent with the calculated values, verifying the validity of the parameter models and the optimization design method. 
Table 3. Experimental results.

\begin{tabular}{|c|c|c|c|c|}
\hline \multicolumn{2}{|c|}{ Parameters } & Calculated & Measured & Error \\
\hline \multicolumn{2}{|c|}{ Leakage inductance@4 kHz $(\mu \mathrm{H})$} & 200 & 209 & $4.5 \%$ \\
\hline \multicolumn{2}{|c|}{ No-load loss (W) } & 200.9 & 208.1 & $3.5 \%$ \\
\hline \multirow{4}{*}{$\begin{array}{c}\text { Temperature } \\
\left({ }^{\circ} \mathrm{C}\right)\end{array}$} & Primary winding & 93.7 & 92 & $1.8 \%$ \\
\hline & Secondary winding & 103.1 & 108.5 & $5.0 \%$ \\
\hline & Main core & 81.3 & 78.2 & $4.0 \%$ \\
\hline & Additional core & 95.9 & 96.8 & $0.9 \%$ \\
\hline
\end{tabular}

\section{Conclusions}

The design and analysis of the inductor-integrated MFT is a challenging multi-physical problem. Facing the application requirements of the DAB converter, the research on the inductor-integrated MFT is carried out. The main conclusions are drawn as follows:

(1) By comparing different integration structures, the CW ELII structure is adopted. The operating mode of CW ELII MFT under typical DAB excitation is analyzed based on the magnetic circuit model, then obtaining the magnetic flux expressions at different phase-shifting duties. Aiming at the characteristics of the irregular cross-section of the main insulation layer and the non-uniform arrangement of conductor layers, the leakage inductance model is improved. The results show that the maximum error of the proposed model is $2.4 \%$, and that of the classical model is $15.0 \%$ for the cases with the non-uniform arrangement of conductor layers.

(2) The thermal network model of air-water combined cooled CW ELII MFT is established. Based on the combination of classical models, the thermal resistance model for the winding air channel under forced convection is proposed, and the relative error with FEM results is less than $10 \%$. The analysis shows that a higher average heat transfer coefficient can be obtained using a higher wind speed or a shorter channel.

(3) The $200 \mathrm{~kW}, 4 \mathrm{kHz}, 200 \mu \mathrm{H}$ MFT for DAB converter is chosen as an example, and the optimal design is carried out using two structures of CW LII and CW ELII. The volume power density of the CW ELII solution is about 130\% higher than that of the CW LII solution with the similar efficiency and cost. The weight power density is $9.9 \%$ higher. A CW ELII MFT prototype was manufactured with the power density of $5.16 \mathrm{~kW} / \mathrm{dm}^{3}$ and the efficiency of $99.30 \%$. The prototype was tested in a 2 MW DC MMC-BDC prototype verifying the electrical and thermal performance.

(4) According to a more extensive comparison and analysis, the power density of the CW LII solution decreases with the increase of the leakage inductance. In contrast, the CW ELII solution achieves a max power density of $5.8 \mathrm{~kW} / \mathrm{dm}^{3}$ when leakage inductance is $20 \%$ (p.u.). The CW ELII solution can achieve a higher power density when the per unit value of leakage inductance exceeds $20 \%$ (p.u.). Both structures achieve the highest efficiency when the leakage inductance is the smallest. The CW ELII solution has an efficiency advantage when the leakage inductance exceeds $40 \%$ (p.u.).

There are still some limitations in this work, which is also the direction of the future research. In this work, the operating frequency of the research object is relatively low. With the popularization of $\mathrm{SiC}$ devices, the operating frequency of MFT is also gradually increasing. Therefore, it is necessary to further develop research of the inductor-integrated MFT with higher operating frequency, focusing on the high-frequency winding loss and the fringing flux loss of the additional core. In this work, only the optimization design of the MFT body is carried out; however, parameters of the converter will significantly affect the MFT design. Therefore, it is necessary to further carry out the system-level optimal design of the converter. The modeling and design method of the CW ELII MFT presented in this paper lays a foundation for further research.

Author Contributions: Conceptualization, X.Z., R.W. and F.X.; Methodology, X.Z. and R.W.; Software, X.Z.; Validation, X.Z.; Writing-original draft preparation, X.Z., W.K. and B.Y. All authors have read and agreed to the published version of the manuscript. 
Funding: This research was funded by the National Natural Science Foundation of China, grant no. 51907199.

Conflicts of Interest: The authors declare no conflict of interest. The funders had no role in the design of the study; in the collection, analyses, or interpretation of data; in the writing of the manuscript, or in the decision to publish the results.

\section{References}

1. Dujic, D.; Zhao, C.; Mester, A.; Steinke, J.K.; Weiss, M.; Lewdeni-Schmid, S.; Chaudhuri, T.; Stefanutti, P. Power electronic traction transformer-low voltage prototype. IEEE Trans. Power Electron. 2013, 28, 5522-5534. [CrossRef]

2. Zhang, L.; Zhang, D.; Shui, H.; Yuan, Y.; Pei, Q.; Zhu, J. Optimization design of medium frequency transformer for the offshore de grid based on multi-objective genetic algorithm. IET Power Electron. 2017, 10, 2157-2162. [CrossRef]

3. Guo, Z.; Yu, R.; Xu, W.; Feng, X.; Huang, A. Design and optimization of a 200-kw medium-frequency transformer for mediumvoltage SiC PV inverters. IEEE Trans. Power Electron. 2021, 36, 10548-10560. [CrossRef]

4. Chen, P.; Xiao, F.; Liu, J.; Zhu, Z.; Ren, Q. Unbalanced Operation Principle and Fast Balancing Charging Strategy of a Cascaded Modular Multilevel Converter-Bidirectional DC-DC Converter in the Shipboard Applications. IEEE Trans. Transp. Electrif. 2020, 6, 1265-1278. [CrossRef]

5. Liu, J.; Zhu, Z.; Xiao, F.; Chen, P.; Ren, Q. A modular three-port DC-DC converter for vessel integrated power system. Trans. China Electrotech. 2020, 35, 4085-4096. [CrossRef]

6. Zhao, B.; Song, Q.; Liu, W.; Sun, Y. Overview of dual-active-bridge isolated bidirectional DC-DC converter of high-frequency-link power-conversion system. IEEE Trans. Power Electron. 2014, 29, 4091-4106. [CrossRef]

7. Yao, P.; Jiang, X.; Xue, P.; Li, S.; Lu, S.; Wang, F. Design optimization of medium frequency transformer for DAB converters with dc bias capacity. IEEE J. Emerg. Sel. Top. Power Electron. 2020, 9, 5043-5054. [CrossRef]

8. Garcia-Bediaga, A.; Villar, I.; Rujas, A.; Mir, L.; Rufer, A. Multiobjective optimization of medium-frequency transformers for isolated soft-switching converters using a genetic algorithm. IEEE Trans. Power Electron. 2017, 32, 2995-3006. [CrossRef]

9. Chen, B. Design optimisation of an inductor-integrated MF transformer for a high-power isolated dual-active-bridge DC-DC converter. IET Power Electron. 2019, 12, 2912-2922. [CrossRef]

10. Chen, B.; Liang, X.; Wan, N. Design methodology for inductor-integrated litz-wired high-power medium-frequency transformer with the nanocrystalline core material for isolated DC-link stage of solid-state transformer. IEEE Trans. Power Electron. 2020, 35, 11557-11573. [CrossRef]

11. Peng, S. Optimal Design of Highly Efficient, Compact and Silent Medium Frequency Transformers for Future Solid-State Transformers. Ph.D. Thesis, ETH Zurich, Zurich, Switzerland, 2017.

12. Villar, I. Multiphysical Characterization of Medium-Frequency Power Electronic Transformers. Ph.D. Thesis, EPFL, Lausanne, Switzerland, 2010.

13. Leibl, M.; Ortiz, G.; Kolar, J. Design and experimental analysis of a medium frequency transformer for solid-state transformer applications. IEEE J. Emerg. Sel. Top. Power Electron. 2017, 5, 110-123. [CrossRef]

14. Zhang, X.; Wang, R.; Xiao, F.; Fan, X.; Yang, G.; Yang, B. Design of highly compact short-time duty power inductors for supercapacitor charging applications. IET Power Electron. 2020, 13, 4377-4385. [CrossRef]

15. Kauder, T.; Belgrand, T.; Lemaître, R.; Thul, A.; Hameyer, K. Medium-frequency power transformer using GOES for a three-phase dual active bridge. J. Magn. Magn. Mater. 2020, 504, 166672. [CrossRef]

16. Marko, M.; Dujic, D. $100 \mathrm{~kW}, 10 \mathrm{kHz}$ medium-frequency transformer design optimization and experimental verification. IEEE Trans. Power Electron. 2019, 34, 1696-1708. [CrossRef]

17. Marko, M.; Drazen, D. Sensitivity Analysis of Medium-Frequency Transformer Designs for Solid-State Transformers. IEEE Trans. Power Electron. 2019, 34, 8356-8367. [CrossRef]

18. Yu, X.; Su, J.; Lai, J.; Guo, S. Analytical optimization of nonsaturated thermally limited high-frequency transformer/inductor design considering discreteness of design variables. IEEE Trans. Power Electron. 2019, 35, 6231-6250. [CrossRef]

19. Cougo, B.; Kolar, J.W. Integration of leakage inductance in tape wound core transformers for dual active bridge converters. In Proceedings of the 7th International Conference on Integrated Power Electronics Systems (CIPS), Nuremberg, Germany, 6-8 March 2012.

20. Venkatachalam, K.; Sullivan, C.; Abdallah, T.; Tacca, H. Accurate prediction of ferrite core loss with nonsinusoidal waveforms using only Steinmetz parameters. In Proceedings of the IEEE Workshop on Computers in Power Electronics, Mayaguez, PR, USA, 3-4 June 2002.

21. Dowell, P. Effects of eddy currents in transformer windings. Proc. Inst. Elect. Eng. 1966, 113, 1387-1394. [CrossRef]

22. Mogorovic, M.; Dujic, D. Medium frequency transformer leakage inductance modeling and experimental verification. In Proceedings of the Energy Conversion Congress and Exposition (ECCE), Cincinnati, OH, USA, 1-5 October 2017.

23. Lv, F.; Guo, Y.; Li, P. Calculation and correction method for leakage inductance of high-power medium-frequency transformer. High Volt. Eng. 2016, 42, 1702-1707. [CrossRef]

24. Mclyman, C.W.T. Transformer and Inductor Design Handbook; China Elect. Power Press: Beijing, China, 2008. 
25. Balakrishnan, A.; Joines, W.; Wilson, T. Air-gap reluctance and inductance calculations for magnetic circuits using a SchwarzChristoffel transformation. IEEE Trans. Power Electron. 1997, 12, 654-663. [CrossRef]

26. Zhang, X.; Xiao, F.; Wang, R.; Fan, X.; Wang, H. Improved Calculation method for inductance value of the air-gap inductor. In Proceedings of the 1st China International Youth Conference on Electrical Engineering (CIYCEE), Wuhan, China, 1-4 November 2020.

27. Incropera, F.; DeWitt, D.; Bergman, T.; Lavine, A. Fundamentals of Heat and Mass Transfer; Chemical Industry Press: Beijing, China, 2007.

28. VDI. VDI Heat Atlas; Springer: Berlin/Heidelberg, Germany, 2010. 